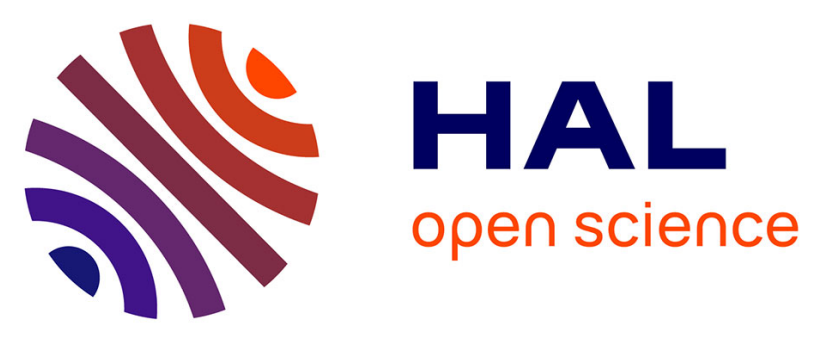

\title{
Micronutrient and Protein Deficiencies After Gastric Bypass and Sleeve Gastrectomy: a one-year Follow-up
} Eric O. Verger, Judith Aron-Wisnewsky, Maria Carlota Dao, Brandon D. Kayser, Jean-Michel Oppert, Jean-Luc Bouillot, Adriana Torcivia, Karine Clément

\section{To cite this version:}

Eric O. Verger, Judith Aron-Wisnewsky, Maria Carlota Dao, Brandon D. Kayser, Jean-Michel Oppert, et al.. Micronutrient and Protein Deficiencies After Gastric Bypass and Sleeve Gastrectomy: a one-year Follow-up. Obesity Surgery, 2016, 26 (4), pp.785-796. 10.1007/s11695-015-1803-7 . hal01297330

\section{HAL Id: hal-01297330 \\ https: / hal.sorbonne-universite.fr/hal-01297330}

Submitted on 4 Apr 2016

HAL is a multi-disciplinary open access archive for the deposit and dissemination of scientific research documents, whether they are published or not. The documents may come from teaching and research institutions in France or abroad, or from public or private research centers.
L'archive ouverte pluridisciplinaire HAL, est destinée au dépôt et à la diffusion de documents scientifiques de niveau recherche, publiés ou non, émanant des établissements d'enseignement et de recherche français ou étrangers, des laboratoires publics ou privés. 


\section{Micronutrient and protein deficiencies after gastric bypass and sleeve gastrectomy: a one-year follow-up}

\section{Original Contribution}

Eric O. Verger $\mathrm{PhD}^{1,2^{*}}$, Judith Aron-Wisnewsky MD, PhD ${ }^{1,2,3^{*}}$, Maria Carlota Dao $\mathrm{PhD}^{1,2}$, Brandon D. Kayser $\mathrm{PhD}^{1,2}$, Jean-Michel Oppert MD, PhD ${ }^{1}$, Jean-Luc Bouillot MD, $\mathrm{PhD}^{4}$, Adriana Torcivia MD ${ }^{5}$, Karine Clément MD, PhD. ${ }^{1,2,3}$

*These authors contributed equally to this work.

${ }^{1}$ Institute of Cardiometabolism and Nutrition, ICAN, Assistance Publique-Hôpitaux de Paris, Pitié-Salpêtrière hospital, Nutrition department, F-75013, Paris, France. ${ }^{2}$ INSERM, UMR_S U1166, NutriOmics team, F-75013, Paris, France. ${ }^{3}$ Sorbonne Universités, UPMC University Paris 06, UMR_S 1166 I, ICAN, Nutriomics team, F-75005, Paris, France. ${ }^{4}$ Assistance Publique-Hôpitaux de Paris, Visceral surgery Department, Ambroise Paré Hospital, 92100 Boulogne-Billancourt, France. ${ }^{5}$ Assistance Publique-Hôpitaux de Paris, Visceral surgery Department, Pitié-Salpêtrière hospital, F-75013, Paris, France

Email addresses: e.verger@ican-institute.org; judith.aron-wisnewsky@psl.aphp.fr; mc.dao@ican-institute.org; b.kayser@ican-institute.org; jean-michel.oppert@psl.aphp.fr; jl.bouillot@apr.aphp.fr; adriana.torcivia@psl.aphp.fr; karine.clement@psl.aphp.fr

\section{Correspondence to:}

Dr. Judith Aron-Wisnewsky and Prof. Karine Clément

Institute of Cardiometabolism and Nutrition

Institut E3M, 83 boulevard de 1'Hôpital,

75013 Paris, France

Email: judith.aron-wisnewsky@psl.aphp.fr

Tel: 33 (0) 142177541

Running title: Malnutrition after surgery

Financial Support: This project is supported by the «Contrat de Recherche Clinique » from Health ministry (CRC Fibrota P100503 - IDRCB 2011-A00759-32, recorded on clinical trial website (NCT: NCT01655017), as well as by the European Union's Seventh Framework Programme for research, technological development and demonstration under grant agreement HEALTH-F4-2012-305312 (Metacardis) and the National Agency of Research (Investissement D'Avenir, ANR-10-IAHU-05). JAW received a grant from Institut Appert and from Nestlé research. The authors declare that they have no conflict of interest.

The authors wish to thank Ms Agathe Arlotti for the coordination of programs on bariatric surgery (BARICAN program), Ms Valentine Lemoine for her help in the follow-up of the patients and Dr Florence Marchelli who contributed to clinical and biological database constitution. 


\section{AbSTRACT}

2 Background: Roux-en-Y gastric bypass (GBP) and sleeve gastrectomy (SG) have increased

3 dramatically, potentially increasing the prevalence of nutritional deficiencies. The aim of this

4 study was to analyze the effects of food restriction during the first year (reviewer \#1, 5 comment \#1) after bariatric surgery (BS) on nutritional parameters.

Methods: 22 and 30 obese patients undergoing GBP and SG were prospectively followed at 7 baseline and three, six and twelve months after $\mathrm{BS}(\mathrm{N}=14$ and $\mathrm{N}=19$ at $\mathrm{T} 12)$ (reviewer \#1, 8 comments \#2\&3). We evaluated food intake and nutrient adequacy (T0, T3, T12), as well as 9 serum vitamins and minerals concentration (T0, T3, T6, T12).

Results: At baseline, GBP and SG patients had similar clinical characteristics, food intake, nutrient adequacy and serum concentration. The drastic energy and food reduction led to very low probabilities of adequacy for nutrients similar in both models (T3, T12). Serum analysis demonstrated a continuous decrease in prealbumin during the follow-up, indicating mild protein depletion in $37 \%$ and $38 \%$ of GBP patients and $57 \%$ and $52 \%$ of SG patients, respectively at T3 and T12 (reviewer \#1, comments \#5,6\&7). Conversely, despite the low probabilities of adequacy observed at $\mathrm{T} 3$ and $\mathrm{T} 12$, systematic multivitamin and mineral supplementation after GBP and SG prevented most nutritional deficiencies.

Conclusion: GBP and SG have comparable effects in terms of energy and food restriction, and subsequent risk of micronutrient and protein deficiencies in the first year post BS. Such results advocate for a cautious monitoring of protein intake after GPB and SG and a systematic multivitamin and mineral supplementation in the first year after SG.

Keywords: Bariatric surgery; Roux-en-Y Gastric Bypass; Sleeve gastrectomy; Protein deficiency; Multivitamin and mineral supplementation. 


\section{INTRODUCTION}

Among the few therapeutic tools to treat morbid obesity, bariatric surgery (BS) appears to be the most effective strategy as demonstrated by its ability to obtain major and sustainable weight loss along with significant improvement of obesity related-comorbidities $[1,2]$. As a result, the number of interventions has dramatically risen worldwide, and Rouxen-Y Gastric Bypass (GBP) and Sleeve Gastrectomy (SG) represented respectively $47 \%$ and $28 \%$ of the 340,000 BS performed in 2011 [3]. Since 2008, SG has emerged to such an extent that it has become the most common procedure in several countries, as is the case in France [3]. Although the two surgical techniques and their mechanisms of action differ, they appear to be equally safe and both induce significant weight loss post-surgery [4].

GBP includes diet restriction as well as the bypass of the proximal part of the jejunum involved in nutrient absorption whereas SG is less invasive and principally restricts the volume of the stomach [5] (reviewer \#2, comment \#4). Therefore SG, compared to GBP, might be viewed as less likely to exacerbate the risk of micronutrient deficiencies in obese patients who are already prone to such deficiencies before surgery [6]. Nevertheless, some studies have demonstrated a considerably higher prevalence of nutrient deficiencies after SG [7-11]. Others, comparing GBP and SG, found quite similar prevalence after both procedures [12-16]. Although study designs differed, these converging results highlight the importance of daily multivitamin and mineral supplementation after both procedures, at least in the first year for SG, in accordance with the latest US guidelines [17]. While the previously mentioned studies evaluated nutrient deficiencies using serum biomarker concentrations, only very few have evaluated food and nutrient intake after GBP and SG: Freeman et al. evaluated food intake two to four years after surgery [18], Moizé et al. and Coupaye et al. evaluated the overall macronutrient intake during one year after BS but did not quantify micronutrient 
intake [15,19], and Moizé et al. evaluated macronutrient and some selected mineral intake 51

during five years after BS [14].

Therefore, we aimed to analyze food restriction effects on the nutritional adequacy of the diet, on macro and micronutrient intake evolution as well as their consequences in terms 54 of bioclinical evolution and micronutrient serum level during one year after both GBP and 55 SG.

\section{Material ANd Methods}

\section{Patients}

Obese candidates for either GBP or SG according to the international bariatric surgery guidelines [20] (i.e. body mass index $(\mathrm{BMI}) \geq 40 \mathrm{~kg} / \mathrm{m}^{2}$, or $\geq 35 \mathrm{~kg} / \mathrm{m}^{2}$ with at least one severe obesity-related comorbidity) were treated in the Obesity Unit of Pitié-Salpetrière Hospital, Institute of Cardio-metabolism and Nutrition, ICAN, Paris, France. Patients determined the choice of technique, and advised by a multidisciplinary panel, from the hospital based on medical history, level of corpulence and obesity-related comorbidity. Weight stable patients were enrolled consecutively in this prospective non-randomized study from January 2012. Hotel-Dieu hospital ethics committee approved the clinical protocol (number P100503 IDRCB 2011-A00759-32) which was recorded on clinical trial website (NCT: NCT01655017). Subjects gave their written informed consent prior to the study inclusion. Medical history and clinical evaluation were obtained at baseline and during the follow-up at three (T3), six (T6) and twelve months (T12) as described elsewhere [21] (reviewer \#1, comment \#1). Anthropometric parameters were estimated by whole-body fanbeam DXA scanning (Hologic Discovery W, software v12.6, 2; Hologic, Bedford, MA), as previously described [22]. Variables from DXA used in the analyses were total and appendicular fat free mass (FFM, in $\mathrm{kg}$ ), and total and appendicular fat mass (FM, in $\mathrm{kg}$ ), 
where appendicular FFM (or FM) was calculated as the sum of FFM (or FM) from both arms

76 and both legs. Basal metabolic rate (BMR) was assessed with indirect calorimetry (Deltatrac II monitor, Datex Instrumentarium Corp., Helsinki, Finland) enabling the evaluation of underreporting of dietary intake [23].

\section{Dietary data and nutrient intakes}

At baseline, T3 and T12, patients completed three consecutive web-based $24 \mathrm{~h}$ dietary records as described elsewhere [24], including two weekdays and one day on the weekend whenever possible. All foods and beverages consumed at breakfast, lunch, dinner and snacks were recorded. Validated photographs enabled patients to estimate portion size for each reported food and beverage item [25]. Patients were also asked to indicate multivitamin and mineral supplements use, specifying the product name and amount, following the nutritional deficiency prevention treatment prescribed for every patient at our center, as described in [26]. This includes supplementation during two weeks before surgery of vitamin D (once

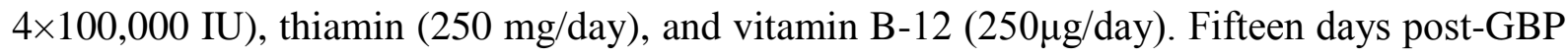
and SG, multivitamin and mineral supplements including Azinc "Forme et vitalité" $($ (two capsules per day, containing $800 \mu \mathrm{g}$ vitamin $\mathrm{A}, 1.4 \mathrm{mg}$ thiamin, $200 \mu \mathrm{g}$ folate, $1 \mu \mathrm{g}$ vitamin $\mathrm{B}$ 12, $120 \mathrm{mg}$ vitamin C, $200 \mathrm{IU}$ vitamin $\mathrm{D}, 8 \mathrm{mg}$ iron and $15 \mathrm{mg}$ zinc), iron ( $2 \times 80 \mathrm{mg} /$ day), vitamin D (800 IU/day), and calcium (1,000mg/day) were started and continued for the first year in both BS procedures. Intake of nutrients derived from food were calculated using an updated version of the French database CIQUAL 2008 [27] which included more than 3,400 different food items. Nutrient intakes from multivitamin and mineral supplements were calculated using nutrient profile based on the product name. Ingested foods were categorized into 4 main food groups when possible: (i) fruit and vegetables, (ii) starchy foods, (iii) dairy products, and (iv) meat and fish. The food groups were defined according to the French 
100 National Nutrition and Health Program [28] and expressed in servings per day based on

\section{Nutrient adequacy of the diet}

Nutrient intake adequacy for each patient was calculated using the PANDiet index [30]. Briefly, probability of adequacy for each nutrient was calculated, ranging from 0 to 1 , where 1 represents a $100 \%$ probability that the usual intake is adequate (i.e. it satisfies the requirement or is not excessive compared to a reference value). According to this definition, the probabilities of adequacy were computed to obtain the Adequacy sub-score (the higher, the better the intake satisfies the nutrient requirements) and the Moderation sub-score (the higher, the less likely the intake is excessive). The PANDiet score is taken as the mean of the Adequacy and Moderation sub-scores, and ranges from 0 to 100; the higher the score, the better the nutrient adequacy of the diet. As reference values, we used French nutritional 113 recommendations for healthy adults or European Union values when specific 114 recommendations were lacking.

116 Biochemical analyses

concentrations of 25(OH)-vitamin-D3 and parathyroid hormone $(\mathrm{PTH})$ were measured by 123 chemiluminescent assay (310600 Liaison XL Diasorin and 11972103 Modular E 170 Roche, 124 respectively), vitamin B-12 and folate were assessed using immunoanalysis ECL sandwich, 
and thiamin and vitamin B-6 were assessed using HPLC [6]. Vitamin and mineral deficiencies

were defined as a result below the lower normal value given by the manufacturer [32].

Secondary hyperparathyroidism was defined as an elevated PTH, above the high normal laboratory value. All measurements were conducted at baseline, T3, T6 and T12 (except for 25(OH)-vitamin-D3, PTH, thiamin, folate and vitamin B-12 at T3, and PTH at T6).

\section{Statistical analyses}

Continuous variables are presented as median and interquartile range (IQR) and frequencies as percentages (reviewer \#2, comment \#2). Mann-Whitney and paired Wilcoxon rank-sum tests were, respectively, used to compare continuous variables between surgical groups and time-points. Chi-squared and $\mathrm{McNemar}$ tests were used to compare frequencies between surgical groups and time-points, respectively. An overall $\alpha$ level of 5\% was used for statistical tests following Holm-Bonferroni correction. These analyses were conducted on both the patients who completed T3 and T6, and on the patients who completed T3, T6 and T12 (reviewer \#1, comment \#1). Since no significant difference was observed between two groups of patients both at baseline, and during the follow-up at T3 and T6, outcomes are merged when presented on tables and figures. All analyses were performed using Statistical Analysis Systems statistical software package version 9.3 (SAS Institute, Cary, NC, SA) (reviewer \#2, comment \#3).

\section{RESULTS}

\section{Clinical characteristics}

147 Fifty-two patients were included in this study (22 GBP and 30 SG). All of them 148 completed the first six months follow-up of this study (T3 and T6), and 33 completed the one 149 year follow-up (T3, T6 and T12; 14 GBP and 19 SG) (reviewer \#1, comment \#1). 
Importantly, the two groups were similar at baseline regarding sex, age, corpulence and body

composition (Table 1). Likewise, the severity of obesity related-comorbidities was similar in the two groups, except for glucose intolerance, which was significantly more prevalent in the GBP group (Table 1).

As expected, BS induced significant weight loss in both surgical techniques, however GBP led to a significantly greater weight loss at T6 and T12 compared to SG (Table 1). More specifically, the total and appendicular FFM (in $\mathrm{kg}$ ) significantly decreased at $\mathrm{T} 3$ and then stabilized at T6 and T12 in the two groups, while the total and appendicular FM (in $\mathrm{kg}$ ) significantly decreased along the one year follow-up in the GBP group whereas it significantly decreased until T6 and then stabilized from T6 to T12 in the SG group (Figure 1). As a result, body composition significantly improved as demonstrated by changes in the percentage of FFM and FM (Table 1). GBP induced a significant improvement of obesityrelated comorbidities (except for high blood pressure (HBP)), whereas SG only led to a significant improvement of dyslipidemia at T6 and T12 (Table 1).

\section{Food and macronutrient intakes}

At baseline, no difference was observed for energy, food or macronutrient intakes between the two groups (Table 2). The BMR values revealed that patients from both groups underreported their caloric intake by $8 \%$.

After both GBP and SG, energy intake drastically decreased at T3 and slightly increased at T12, although not reaching baseline intake levels (significant at all time points, Table 2). These changes in energy intake were explained by a significant decrease in food intake at T3 in the two surgical groups (non-significant for dairy products) and a tendency for a modest increase in food intake at T12 (significant for starchy foods in the SG group, Table 2). Total protein intake drastically and significantly decreased at $\mathrm{T} 3$ in both groups, and a 
majority of patients reported protein intake below the recommended value of $60 \mathrm{~g} /$ day $(85.7 \%$

176 after GBP and $79 \%$ after SG, Table 2). Afterwards, total protein intake slightly but 177 significantly increased at T12, although it remained below the baseline levels (Table 2). 178 Furthermore, $61 \%$ of the patients reported low dietary protein intake $(64 \%$ and $58 \%$ 179 respectively for GBP and SG groups, Table 2) at T12. No significant changes in 180 macronutrient distribution (total fat, SFA, PUFA and total carbohydrates) were observed 181 during the follow-up in the two groups (T3 and T12, Table 2). Energy, food and 182 macronutrient intakes were not different between the two groups during the follow-up (T3 183 and T12, Table 2).

\section{Nutrient adequacy of the diet}

At baseline, neither the PANDiet scores nor the probabilities of nutrient adequacy differed between the two groups (Table 3). Low probabilities of adequacy for protein were observed in both groups compared to the French adult population [30].

After both BS, the percentage of patients taking the prescribed systematic multivitamin and mineral supplements significantly increased, from baseline to T3: $14 \%$ versus $77 \%$ for GBP and $10 \%$ vs. $76.7 \%$ for $\mathrm{SG}$, as expected from the recommendations (Table 3). This high adherence was maintained at T12 with $86 \%$ and $68 \%$ respectively for GBP and SG (Table 3). Due to the supplementation, the global nutrient adequacy of the diet did not drop and rather stabilized along the follow-up (PANDiet score and Adequacy subscore were not significantly different at all time points) and the probability of adequacy for vitamin D was improved (Table 3). Of note, when the global nutrient adequacy of the diet was calculated without taking into account the prescribed supplementation, we found that it drastically decreased at T3 and barely increased at T12 (Supplemental Table 1). However, since the prescribed supplementation neither contains protein, fiber nor phosphorus, lower 
200 probabilities of adequacy for these nutrients were observed in both groups at T3 compared to

baseline (Table 3). Furthermore, although the probabilities of adequacy for these four nutrients significantly increased at T12 in both groups compared to T3 due to the slight increase in food intake, they remained below the baseline values (except for protein in the GBP group, Table 3).

\section{Nutritional deficiencies}

At baseline, none of the metabolic and nutritional parameters were different between the two groups (Table 4). As expected in severe obesity, $100 \%$ and $83 \%$ of the patients from the GBP and SG groups, respectively, presented 25(OH)-vitamin-D3 deficiency as seen by serum concentrations below $30 \mathrm{ng} / \mathrm{ml}$ (Table 4) with subsequent secondary hyperparathyroidism in $50 \%$ of the subjects, showing major deficiency in this population.

After both BS, prealbumin concentration drastically and significantly decreased at T3 and further stabilized at T6 and T12 (Table 4). At T12, 38\% of GBP patients and 52\% of SG patients presented mild protein depletion as shown by prealbumin concentration below the normal range of $0.2 \mathrm{~g} / \mathrm{l}$ and $21 \%$ of GBP patients and $16 \%$ of SG patients presented risk of mild protein malnutrition as shown by albumin concentration below the normal value of 37 g/l (Table 4). Of note, two patients in the GBP group and one patient in the SG group presented both mild protein depletion and risk of mild protein malnutrition (reviewer \#1, comments \#5,6\&7). After both BS, vitamin D supplementation enabled a significant increase in 25(OH)-vitamin-D3 serum concentrations at T6, which stabilized at T12 (Table 4). However, $50 \%$ and $21 \%$ of GBP and SG patients, respectively, still displayed secondary hyperparathyroidism at T12 (Table 4). Since all patients were prescribed multivitamin and mineral supplementation, we verified whether this supplementation might induce serum concentrations of selected vitamins and minerals above the normal range at T12. In fact, there 
were only a few such cases in the overall cohort: one with elevated serum thiamin (700 nmol/l) and one with high serum ferritin $(740 \mu \mathrm{g} / \mathrm{l})$ in the SG group, and one with elevated vitamin B12 (580 pmol/l) in the GBP group. Importantly, all such elevations remained below toxic levels.

\section{DisCUSSION}

To the best of our knowledge, this is the first study to assess the relationship between food intake, nutrient adequacy of the diet and nutritional biological parameters systematically measured before, three and twelve months after GBP and SG. In this study where the patients had similar clinical characteristics at baseline (except for T2D prevalence), our main findings are: (i) protein intake significantly decreases after both GBP and SG, inducing mild protein depletion in more than a third of the patients one year after both surgical techniques (reviewer \#1, comments \#5,6\&7); (ii) even though patients after GBP experienced greater weight loss than after SG, both types of surgery induced similar food restriction effects on the nutritional adequacy of the diet and, (iii) systematic multivitamin and mineral supplementation after SG seems to prevent these nutritional deficiencies, the same way as in GBP in the first year.

After one year, we observed that GBP led to significantly greater weight loss compared to $\mathrm{SG}$, in accordance with previous data from the literature, including a large multicenter study [33,34]. Nevertheless, some controversy remains. Indeed other reports show that changes in body weight were similar one year after both GBP and SG $[15,19,35]$, although these were smaller cohorts. We evaluated the evolution of body composition and observed that, in both surgeries, total FFM decreased until three months and then stabilized, 247 whereas total FM displayed a continuous decrease during the follow-up. Our results are 248 consistent with previous reports showing changes in body composition following GBP [22] or 249 SG [36] as measured by DXA. Our results are also concordant with the only study comparing 
250 these outcomes after both sleeve and by-pass [19]. In that study, the continuous weight loss

during one year was due to the decrease of total FM, the total FFM being spared after four months [19]. More importantly, we observed that appendicular FFM decreased until three months and then stabilized, whereas appendicular FM continued to decrease throughout the follow-up period in both models. Appendicular FFM represents a better surrogate of muscle mass than total FFM [37], and this is the first time that this outcome and its evolution have been studied after SG. Interestingly, the change in appendicular FFM was similar in the two surgical procedures.

After both BS, we observed that $61 \%$ of the patients reported daily protein consumption under the recommended value of $60 \mathrm{~g} / \mathrm{day}$ at T12 (64\% for GBP and 58\% for SG). Our results are consistent with those of Andreu et al. and Moizé et al. who found that respectively $37 \%$ and $46 \%$ of patients had a daily protein intake below $60 \mathrm{~g} /$ day one year after BS $[19,38]$. In accordance with those findings, we did not find any difference between GBP and SG [38]. We report a prevalence of insufficient protein intake that is nearly 2-fold higher than that reported by Moizé et al. (61\% versus 37\%), which is mostly attributable to the systematic protein supplementation prescribed by these authors to all of their patients [38]. One objective of recommending a minimal protein intake of $60 \mathrm{~g} /$ day after both GBP and SG is to mitigate post-surgical FFM loss in the first months [17]. Indeed, Moizé et al. demonstrated that patients with insufficient protein intake during the follow-up lost more FFM in both SG and GBP than patients with sufficient protein intake [19]. Because skeletal muscle is the primary site of insulin-stimulated glucose disposal during euglycemia [39], loss of FFM might contribute to the development of insulin resistance and should be avoided in order to maintain the beneficial metabolic outcomes. An important goal of future long-term follow-up studies will be to determine whether insufficient protein intake following BS might 
result in loss of muscular strength. Furthermore, longer-term weight stabilization (and regain) should also be assessed in link with the quantity of protein intake.

After both BS, we also observed that prealbumin concentration significantly decreased, resulting in more than a third of patients exhibiting mild protein depletion (reviewer \#1, comments \#5,6\&7). Our results are in line with the few studies that reported changes in prealbumin concentration after GBP or SG. All studies found lower values at T12 after GBP compared to baseline $[15,40,41]$. Results with SG are more heterogeneous, with reports showing both lower [42] or no change in prealbumin concentrations [15,41]. Of note, Moizé et al. reported that $14 \%$ of GBP and $16 \%$ of SG patients experienced abnormalities in prealbumin concentrations at T12 after BS [14]. As mentioned above, this difference may be due to the systematic prescription of protein supplement in the Hospital Clinic of Barcelona $[19,38]$. Adequate protein intake after BS is of utmost importance to prevent the patients from experiencing hair loss, poor wound healing and adverse effects such as infections after skin repair surgery and ultimately - but rarely - protein-calorie malnutrition [43,44] (reviewer \#1, comments \#5,6\&7).

Although SG merely restricts the volume of the stomach without intestinal malabsorption [5], it also leads to an accelerated gastric emptying (reviewer \#2, comment \#4). Subsequently faster gastrointestinal passage might promote nutrient deficiencies [45], as observed in a recent study with increased faecal excretion of fatty acids [46], resulting in a state of moderate malabsorption. Furthermore, SG decreases gastric intrinsic factor and gastric acid production, two factors involved in vitamin B-12 and iron absorption. Because most of our patients took the prescribed daily multivitamin and mineral supplements one year after both GBP and SG, few patients experienced nutritional abnormalities (except for 25(OH)-vitamin-D3) and there was no difference between the two surgical groups. Our results were consistent with previous data from the literature $[14,15]$. Conversely, others 
reported a higher risk of vitamin $\mathrm{B}-12$ and $25(\mathrm{OH})$-vitamin-D3 deficiencies after GBP

compared to SG [12]. It should be noted that in these three studies, patients undergoing GBP or SG were instructed to take multivitamin and mineral supplements on a daily basis after BS. Another point to take into account, is the risk of developing undesirably high levels of micronutrient concentrations due to the systematic supplementation as was previously reported after SG $[7,8,11]$. Herein, we only identified one patient with serum thiamin and another with serum ferritin above normal range. Nevertheless, it should be noted that the risk of excessive levels in those studies were mostly observed for vitamin A and B-6, which we did not assess. Altogether, these data highlight the importance to prescribe daily multivitamin and mineral supplements after both GBP and SG at least in the first year, but also to monitor the adherence of the patients to their supplementation.

At baseline, the higher prevalence of glucose intolerance in patients undergoing GBP reflects the process of selection for different BS techniques, where GBP is the first choice for patients with T2D or glucose intolerance since it demonstrated its superiority over SG to improve glycemic status post-surgery [35]. We also observed that neither GBP nor SG enabled a significant improvement of HBP in terms of overall prevalence. Nevertheless, both the number of patients treated and the number of treatments per patients tended to decrease after both surgeries, suggesting slight improvement of HBP in this cohort of obese patients with many comorbidities. Nevertheless our data are in accordance with previous studies, which indicated that HBP may not be the best resolved comorbidity after surgery $[47,48]$ (reviewer \#1, comment \#4).

One of the main strengths of our study is the use of a validated web-based method of dietary assessment which allowed us to provide detailed quantification of the food and nutrient intake for each patient [24]. This method allows us to assess the use of multivitamin and mineral supplements and measure adherence of the patients to the supplementation. 
324 Although the interventions were not randomized in our study, our participants had

comparable clinical characteristics at baseline (except for T2D) and were provided the same systematic supplementation regardless of the surgical procedure. The main limitation concerns the relative small number of patients, especially in the group who completed the one year follow-up (reviewer \#1, comment \#1). This may have prevented us from detecting changes between FFM loss and low protein intake after both procedures. Future studies with longer follow-up periods and larger sample size are needed to determine how poor dietary habits and nutritional deficiencies correlate with weight maintenance at longer term and with the improvement or resolution of obesity related co-morbidities. We intend to follow this cohort in the second year of their surgery to assess their evolution in terms of nutritional risks and body composition (reviewer \#2, comment \#7).

In conclusion, we observed similar food restriction effects on the nutritional adequacy of the diet in the first year post GBP and SG surgery. We also observed comparable consequences in terms of bioclinical evolution and micronutrient serum concentrations. Altogether, our results advocate for a cautious monitoring of protein intake and a systematic multivitamin and mineral supplementation after both GPB and SG - at least in the first year for SG.

342 Conflict of interest. The authors declare that they have no conflict of interest. 


\section{REFERENCES}

1. Buchwald H, Avidor Y, Braunwald E, et al. Bariatric surgery: a systematic review and meta-analysis. JAMA. 2004 Oct 13;292(14):1724-37.

2. Mingrone G, Panunzi S, De Gaetano A, et al. Bariatric surgery versus conventional medical therapy for type 2 diabetes. N Engl J Med. 2012 Apr 26;366(17):1577-85.

3. Buchwald H, Oien DM. Metabolic/bariatric surgery worldwide 2011. Obes Surg. 2013 Apr;23(4):427-36.

4. Chang S-H, Stoll CRT, Song J, et al. The effectiveness and risks of bariatric surgery: an updated systematic review and meta-analysis, 2003-2012. JAMA Surg. 2014 Mar;149(3):275-87.

5. Jacobs M, Bisland W, Gomez E, et al. Laparoscopic sleeve gastrectomy: a retrospective review of 1- and 2-year results. Surg Endosc. 2009 Aug 19;24(4):781-5.

6. Aasheim ET, Hofs $\varnothing$ D, Hjelmesaeth J, et al. Vitamin status in morbidly obese patients: a cross-sectional study. Am J Clin Nutr. 2008 Feb;87(2):362-9.

7. Aarts EO, Janssen IMC, Berends FJ. The gastric sleeve: losing weight as fast as micronutrients? Obes Surg. 2011 Feb;21(2):207-11.

8. Damms-Machado A, Friedrich A, Kramer KM, et al. Pre- and postoperative nutritional deficiencies in obese patients undergoing laparoscopic sleeve gastrectomy. Obes Surg. 2012 Jun;22(6):881-9.

9. Pech N, Meyer F, Lippert H, et al. Complications and nutrient deficiencies two years after sleeve gastrectomy. BMC Surg. 2012;12:13.

10. Saif T, Strain GW, Dakin G, et al. Evaluation of nutrient status after laparoscopic sleeve gastrectomy 1, 3, and 5 years after surgery. Surg Obes Relat Dis Off J Am Soc Bariatr Surg. 2012 Oct;8(5):542-7. 
11. Van Rutte PWJ, Aarts EO, Smulders JF, et al. Nutrient deficiencies before and after sleeve gastrectomy. Obes Surg. 2014 Oct;24(10):1639-46.

12. Gehrer S, Kern B, Peters T, et al. Fewer nutrient deficiencies after laparoscopic sleeve gastrectomy (LSG) than after laparoscopic Roux-Y-gastric bypass (LRYGB)-a prospective study. Obes Surg. 2010 Apr;20(4):447-53.

13. Kehagias I, Karamanakos SN, Argentou M, et al. Randomized clinical trial of laparoscopic Roux-en-Y gastric bypass versus laparoscopic sleeve gastrectomy for the management of patients with BMI < $50 \mathrm{~kg} / \mathrm{m} 2$. Obes Surg. $2011 \mathrm{Nov} ; 21(11): 1650-6$.

14. Moizé V, Andreu A, Flores L, et al. Long-term dietary intake and nutritional deficiencies following sleeve gastrectomy or Roux-En-Y gastric bypass in a mediterranean population. J Acad Nutr Diet. 2013 Mar;113(3):400-10.

15. Coupaye $\mathrm{M}$, Rivière $\mathrm{P}$, Breuil $\mathrm{MC}$, et al. Comparison of nutritional status during the first year after sleeve gastrectomy and Roux-en-Y gastric bypass. Obes Surg. 2014 Feb;24(2):276-83.

16. Kwon Y, Kim HJ, Lo Menzo E, et al. Anemia, iron and vitamin B12 deficiencies after sleeve gastrectomy compared to Roux-en-Y gastric bypass: a meta-analysis. Surg Obes Relat Dis Off J Am Soc Bariatr Surg. 2014 Aug;10(4):589-97.

17. Mechanick JI, Youdim A, Jones DB, et al. Clinical Practice Guidelines for the Perioperative Nutritional, Metabolic, and Nonsurgical Support of the Bariatric Surgery Patient - 2013 Update: Cosponsored by American Association of Clinical Endocrinologists, The Obesity Society, and American Society for Metabolic \& Bariatric Surgery. Obes Silver Spring Md. 2013 Mar;21(0 1):S1-27.

18. Freeman RA, Overs SE, Zarshenas N, et al. Food tolerance and diet quality following adjustable gastric banding, sleeve gastrectomy and Roux-en-Y gastric bypass. Obes Res Clin Pract. 2014 Apr;8(2):e115-200. 
19. Moizé V, Andreu A, Rodríguez L, et al. Protein intake and lean tissue mass retention following bariatric surgery. Clin Nutr Edinb Scotl. 2013 Aug;32(4):550-5.

20. Fried M, Yumuk V, Oppert J-M, et al. Interdisciplinary European Guidelines on metabolic and bariatric surgery. Obes Facts. 2013;6(5):449-68.

21. Abdennour M, Reggio S, Le Naour G, et al. Association of adipose tissue and liver fibrosis with tissue stiffness in morbid obesity: links with diabetes and BMI loss after gastric bypass. J Clin Endocrinol Metab. 2014 Mar;99(3):898-907.

22. Ciangura C, Bouillot J-L, Lloret-Linares C, et al. Dynamics of change in total and regional body composition after gastric bypass in obese patients. Obes Silver Spring Md. 2010 Apr;18(4):760-5.

23. Nielsen BM, Nielsen MM, Toubro S, et al. Past and current body size affect validity of reported energy intake among middle-aged Danish men. J Nutr. 2009 Dec;139(12):233743.

24. Touvier M, Kesse-Guyot E, Méjean C, et al. Comparison between an interactive webbased self-administered $24 \mathrm{~h}$ dietary record and an interview by a dietitian for large-scale epidemiological studies. Br J Nutr. 2011 Apr;105(7):1055-64.

25. Le Moullec N, Deheeger M, Preziosi P, et al. Validation du manuel-photos utilisé pour l'enquête alimentaire de l'étude SU.VI.MAX. Cah Nutr Diététique. 31(3):158-64.

26. Gesquiere I, Aron-Wisnewsky J, Foulon V, et al. Medication cost is significantly reduced after Roux-en-Y gastric bypass in obese patients. Obes Surg. 2014 Nov;24(11):1896903.

27. Afssa - Table de composition nutritionnelle des aliments CIQUAL 2008 (French Food Composition Table - CIQUAL 2008). Available from:

https://pro.anses.fr/tableciqual/index.htm. 
28. Hercberg S, Chat-Yung S, Chaulia M. The French National Nutrition and Health Program: 2001-2006-2010. Int J Public Health. 2008;53(2):68-77.

29. Estaquio C, Kesse-Guyot E, Deschamps V, et al. Adherence to the French Programme National Nutrition Santé Guideline Score is associated with better nutrient intake and nutritional status. J Am Diet Assoc. 2009 Jun;109(6):1031-41.

30. Verger EO, Mariotti F, Holmes BA, et al. Evaluation of a diet quality index based on the probability of adequate nutrient intake (PANDiet) using national French and US dietary surveys. PloS One. 2012;7(8):e42155.

31. Aron-Wisnewsky J, Minville C, Tordjman J, et al. Chronic intermittent hypoxia is a major trigger for non-alcoholic fatty liver disease in morbid obese. J Hepatol. 2012 Jan;56(1):225-33.

32. Ledoux S, Msika S, Moussa F, et al. Comparison of nutritional consequences of conventional therapy of obesity, adjustable gastric banding, and gastric bypass. Obes Surg. 2006 Aug;16(8):1041-9.

33. Hutter MM, Schirmer BD, Jones DB, et al. First report from the American College of Surgeons Bariatric Surgery Center Network: laparoscopic sleeve gastrectomy has morbidity and effectiveness positioned between the band and the bypass. Ann Surg. 2011 Sep;254(3):410-420; discussion 420-422.

34. Lim DM, Taller J, Bertucci W, et al. Comparison of laparoscopic sleeve gastrectomy to laparoscopic Roux-en-Y gastric bypass for morbid obesity in a military institution. Surg Obes Relat Dis Off J Am Soc Bariatr Surg. 2014 Apr;10(2):269-76.

35. Kashyap SR, Bhatt DL, Wolski K, et al. Metabolic effects of bariatric surgery in patients with moderate obesity and type 2 diabetes: analysis of a randomized control trial comparing surgery with intensive medical treatment. Diabetes Care. 2013 Aug;36(8):2175-82. 
36. Bužga M, Zavadilová V, Holéczy $\mathrm{P}$, et al. Dietary intake and ghrelin and leptin changes after sleeve gastrectomy. Videosurgery Miniinvasive Tech. 2014 Dec;9(4):554-61.

37. Kim J, Wang Z, Heymsfield SB, et al. Total-body skeletal muscle mass: estimation by a new dual-energy X-ray absorptiometry method. Am J Clin Nutr. 2002 Aug 1;76(2):37883.

38. Andreu A, Moizé V, Rodríguez L, et al. Protein intake, body composition, and protein status following bariatric surgery. Obes Surg. 2010 Nov;20(11):1509-15.

39. DeFronzo RA, Jacot E, Jequier E, et al. The effect of insulin on the disposal of intravenous glucose. Results from indirect calorimetry and hepatic and femoral venous catheterization. Diabetes. 1981 Dec;30(12):1000-7.

40. Coupaye M, Puchaux K, Bogard C, et al. Nutritional consequences of adjustable gastric banding and gastric bypass: a 1-year prospective study. Obes Surg. 2009 Jan;19(1):5665.

41. Sallé A, Demarsy D, Poirier AL, et al. Zinc deficiency: a frequent and underestimated complication after bariatric surgery. Obes Surg. 2010 Dec;20(12):1660-70.

42. Friedrich AE, Damms-Machado A, Meile T, et al. Laparoscopic sleeve gastrectomy compared to a multidisciplinary weight loss program for obesity--effects on body composition and protein status. Obes Surg. 2013 Dec;23(12):1957-65.

43. Agha-Mohammadi S, Hurwitz DJ. Nutritional deficiency of post-bariatric surgery body contouring patients: what every plastic surgeon should know. Plast Reconstr Surg. 2008 Aug;122(2):604-13.

44. Faria SL, Faria OP, Buffington C, de Almeida Cardeal M, Ito MK. Dietary protein intake and bariatric surgery patients: a review. Obes Surg. 2011 Nov;21(11):1798-805.

45. Melissas J, Daskalakis M, Koukouraki S, et al. Sleeve Gastrectomy - A "Food Limiting" Operation. Obes Surg. 2008 Jul 29;18(10):1251-6. 
46. Damms-Machado A, Mitra S, Schollenberger AE, et al. Effects of Surgical and Dietary Weight Loss Therapy for Obesity on Gut Microbiota Composition and Nutrient Absorption. BioMed Res Int. 2015 Feb 1;2015:e806248.

47. Sjöström L, Narbro K, Sjöström CD, et al. Effects of bariatric surgery on mortality in Swedish obese subjects. Engl J Med. 2007 Aug 23;357(8):741-52.

48. Gesquiere I, Aron-Wisnewsky J, Foulon V, et al. Medication cost is significantly reduced after Roux-en-Y gastric bypass in obese patients. Obes Surg. 2014 Nov;24(11):1896-903. 


\section{TABLES}

TABLE 1. Anthropometric parameters and clinical characteristics according the surgical models at baseline, 3 months, 6 months and 12 months ${ }^{1}$.

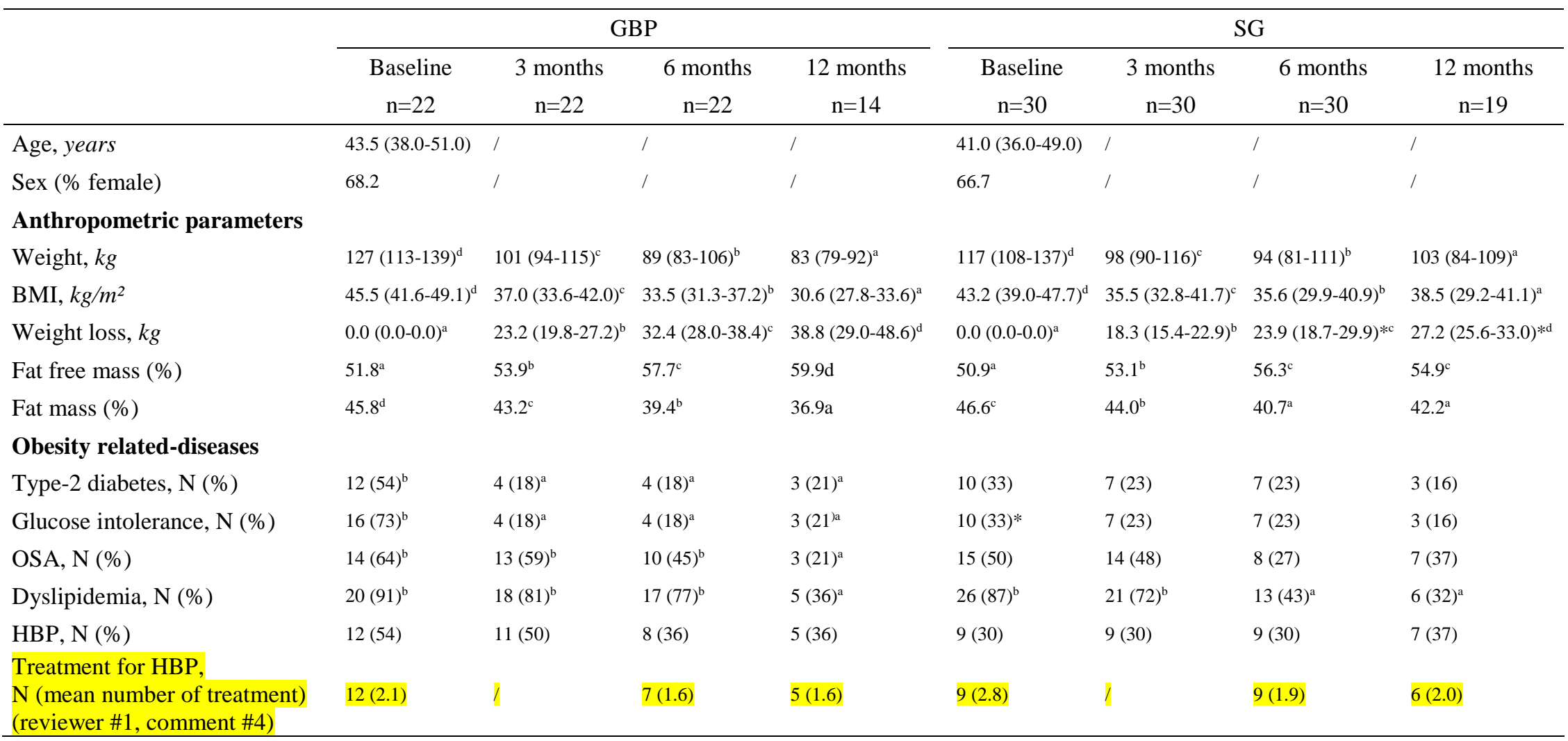

${ }^{1}$ Labeled medians or percentages without a common letter differ between time-points for each surgical model, as tested by paired pairwise post hoc comparisons with Holm-Bonferroni correction or paired McNemar test. *Represents significant differences between GBP and SG. Glucose intolerance is defined as either fasting hyperglycemia $(\mathrm{lg} / \mathrm{l} \leq G<1.26 \mathrm{~g} / \mathrm{l})$ or $6 \% \leq H B A 1 c<6.5 \%)$; dyslipidemia is defined as a patient with treatment (statin or fibrate) or hypertriglyceridemia $\geq 1.5 \mathrm{~g} / \mathrm{l}$ or hypoHDL $<0.5 \mathrm{~g} / \mathrm{l}$ for women and hypoHDL $<0.4 \mathrm{~g} / \mathrm{l}$ for men; High blood pressure 
$(H B P)$ is defined as a systolic blood pressure >140 mmHg and/or a diastolic blood pressure >90mmHg or patients with an anti-hypertensive treatment; obstructive sleep apnea (OSA) is defined as an Index Apnea Hypopnea >5/hour with or without treatment.) 
TABLE 2. Energy, food and macronutrient intakes according to the surgical models at baseline, 3 months and 12 months $^{1}$.

\begin{tabular}{|c|c|c|c|c|c|c|}
\hline & \multicolumn{3}{|c|}{ GBP } & \multicolumn{3}{|c|}{ SG } \\
\hline & $\begin{array}{c}\text { Baseline } \\
n=22\end{array}$ & $\begin{array}{c}3 \text { months } \\
n=22\end{array}$ & $\begin{array}{c}12 \text { months } \\
n=14\end{array}$ & $\begin{array}{c}\text { Baseline } \\
n=30\end{array}$ & $\begin{array}{c}3 \text { months } \\
n=30\end{array}$ & $\begin{array}{c}12 \text { months } \\
\mathrm{n}=19\end{array}$ \\
\hline \multicolumn{7}{|l|}{ Energy and food intakes } \\
\hline Energy intake, $\mathrm{kcal} / \mathrm{d}$ & $2005(1539-2266)^{\mathrm{c}}$ & $711(615-1006)^{\mathrm{a}}$ & $1226(8133-1559)^{\mathrm{b}}$ & $1658(1445-2395)^{\mathrm{c}}$ & $833(539-1108)^{\mathrm{a}}$ & $1078(793-1354)^{b}$ \\
\hline $\mathrm{BMR}, \mathrm{kcal} / \mathrm{d}$ & $2179(2005-2409)^{\mathrm{c}}$ & $1770(1702-2072)^{b}$ & $1653(1480-1791)^{\mathrm{a}}$ & $1959(1853-2218)^{\mathrm{c}}$ & $1742(1593-1894)^{b}$ & $1686(1565-1963)^{\mathrm{a}}$ \\
\hline Fruit and vegetables, serving/d & $4.8(3.2-7.0)^{\mathrm{b}}$ & $2.2(0.8-3.2)^{\mathrm{a}}$ & $2.1(1.5-3.9)^{\mathrm{ab}}$ & $3.0(1.6-4.3)^{\mathrm{b}}$ & $1.5(0.8-2.1)^{\mathrm{a}}$ & $1.4(1.0-2.6)^{\mathrm{ab}}$ \\
\hline Starchy foods, serving/d & $2.8(2.1-3.7)^{\mathrm{b}}$ & $0.7(0.3-1.2)^{\mathrm{a}}$ & $1.1(0.8-1.6)^{\mathrm{a}}$ & $2.6(2.1-3.3)^{\mathrm{c}}$ & $0.7(0.3-1.1)^{\mathrm{a}}$ & $1.2(0.7-1.7)^{\mathrm{b}}$ \\
\hline Dairy products, serving/d & $2.1(1.3-3.1)$ & $1.7(0.5-2.6)$ & $2.1(0.8-2.5)$ & $1.6(1.0-2.4)$ & $1.4(0.6-1.9)$ & $1.2(0.7-1.7)$ \\
\hline Meat and fish, serving/d & $1.4(1.0-2.6)^{\mathrm{b}}$ & $0.8(0.6-1.1)^{\mathrm{a}}$ & $0.7(0.4-1.6)^{\mathrm{ab}}$ & $1.6(1.1-2.5)^{\mathrm{b}}$ & $0.9(0.6-1.4)^{\mathrm{a}}$ & $1.0(0.7-1.8)^{\mathrm{ab}}$ \\
\hline \multicolumn{7}{|l|}{ Macronutrient intakes } \\
\hline Protein, $g / d$ & $83.5(70.6-105.6)^{\mathrm{c}}$ & $41,7(24,0-49,0)^{\mathrm{a}}$ & $50,4(36,9-65,2)^{\mathrm{b}}$ & $78,3(64.0-107,2)^{\mathrm{c}}$ & $41,2(26,8-52,6)^{\mathrm{a}}$ & $51,8(36,4-65,3)^{\mathrm{b}}$ \\
\hline $\mathrm{N}(\%)<60 \mathrm{~g} / d$ & $2(9)^{\mathrm{a}}$ & $19(86)^{b}$ & $9(64)^{\mathrm{b}}$ & $4(13)^{\mathrm{a}}$ & $26(87)^{b}$ & $11(58)^{\mathrm{b}}$ \\
\hline Protein, $g / \mathrm{kg} / \mathrm{d}$ & $0.66(0.57-0.73)^{\mathrm{b}}$ & $0,38(0,24-0,46)^{\mathrm{a}}$ & $0,59(0,48-0,715)^{\mathrm{b}}$ & $0,65(0,57-0,80)^{\mathrm{c}}$ & $0,39(0,29-0,50)^{\mathrm{a}}$ & $0,46(0,39-0,74)^{\mathrm{b}}$ \\
\hline Total Lipid, \%EI/d & $32.0(30.0-40.6)$ & $36,8(32,4-39,3)$ & $38,8(33,6-45,6)$ & $37,4(33,2-39,9)$ & $41,6(35,8-44,7)$ & $39,5(37,1-44,5)$ \\
\hline $\mathrm{SFA}, \% E I / d$ & $14.7(11.3-16.4)$ & $15,5(13,1-16,6)$ & $17,4(13,7-20,9)$ & $15,6(14,5-18,7)$ & $17,4(15,3-19,6)$ & $15,8(13,7-19,4)$ \\
\hline PUFA, $\% E I / d$ & $4.8(4.2-5.8)$ & $4,3(3,2-6,4)$ & $3,5(3,0-5,5)$ & $5.0(4,0-5,9)$ & $5,0(3,3-6,4)$ & $5,6(4,3-8.0)$ \\
\hline Total Carbohydrate, $\% E I / d$ & $47.8(42.0-49.7)$ & $44.0(38.9-49.2)$ & $42,2(35,4-47,1)$ & $44.1(40.0-46.7)$ & $37.4(32.3-46.8)$ & $42,4(33,4-45,1)$ \\
\hline
\end{tabular}

${ }^{1}$ Labeled medians or percentages without a common letter differ between time-points for each surgical model, as tested by paired pairwise post hoc comparisons with Holm-Bonferroni correction or paired McNemar test. 
TABLE 3. Multivitamin and mineral supplementation, PANDiet scores and probabilities of nutrient adequacy according to the surgical models at baseline, 3 months and 12 months ${ }^{1}$

\begin{tabular}{|c|c|c|c|c|c|c|}
\hline & \multicolumn{3}{|c|}{ GBP } & \multicolumn{3}{|c|}{ SG } \\
\hline & $\begin{array}{c}\text { Baseline } \\
n=22\end{array}$ & $\begin{array}{c}3 \text { months } \\
n=22\end{array}$ & $\begin{array}{c}12 \text { months } \\
n=14\end{array}$ & $\begin{array}{c}\text { Baseline } \\
n=30\end{array}$ & $\begin{array}{c}3 \text { months } \\
n=30\end{array}$ & $\begin{array}{c}12 \text { months } \\
n=19\end{array}$ \\
\hline Supplementation, N (\%) & $3(14)^{\mathrm{a}}$ & $17(77)^{b}$ & $12(86)^{b}$ & $3(10)^{\mathrm{a}}$ & $23(77)^{b}$ & $13(68)^{b}$ \\
\hline PANDiet & $67.4(60.7-70.7)$ & $74.7(61.5-76.3)$ & $71.0(65.3-75.0)$ & $57.7(54.0-63.1)$ & $65.3(57.2-71.3)$ & $65.0(57.4-73.0)$ \\
\hline Moderation Sub-score & $64.6(58.6-85.6)$ & $82.1(75.6-85.3)$ & $74.3(61.8-81.2)$ & $63.2(51.3-73.5)^{\mathrm{a}}$ & $70.9(63.83-80.0)^{\mathrm{b}}$ & $73.1(66.7-78.1)^{\mathrm{b}}$ \\
\hline Protein & $1.00(1.00-1.00)$ & $1.00(1.00-1.00)$ & $1.00(1.00-1.00)$ & $1.00(1.00-1.00)$ & $1.00(1.00-1.00)$ & $1.00(1.00-1.00)$ \\
\hline Total Carbohydrate & $1.00(1.00-1.00)$ & $1.00(0.97-1.00)$ & $1.00(0.98-1.00)$ & $1.00(1.00-1.00)$ & $1.00(1.00-1.00)$ & $1.00(1.00-1.00)$ \\
\hline Total Fat & $0.99(.035-1.00)$ & $0.81(0.58-0.93)$ & $0.59(0.05-0.95)$ & $0.84(0.52-0.96)$ & $0.42(0.02-0.84)$ & $0.57(0.05-0.89)$ \\
\hline SFA & $0.19(0.04-0.61)$ & $0.12(0.02-0.35)$ & $0.02(0.00-0.29)$ & $0.05(0.00-0.30)$ & $0.02(0.00-0.15)$ & $0.10(0.00-0.28)$ \\
\hline Cholesterol & $0.66(0.36-0.99)^{\mathrm{a}}$ & $0.99(0.94-1.00)^{\mathrm{b}}$ & $0.95(0.51-0.99)^{\mathrm{ab}}$ & $0.41(0.15-0.79)^{\mathrm{a}}$ & $0.98(0.65-1.00)^{\mathrm{b}}$ & $1.00(0.65-1.00)^{\mathrm{b}}$ \\
\hline Sodium & $0.43(0.18-0.77)^{\mathrm{a}}$ & $1.00(0.96-1.00)^{\mathrm{c}}$ & $0.97(0.71-1.00)^{\mathrm{b}}$ & $0.51(0.01-0.78)^{\mathrm{a}}$ & $1.00(0.97-1.00)^{\mathrm{b}}$ & $0.99(0.70-1.00)^{\mathrm{b}}$ \\
\hline Adequacy Sub-score & $63.7(53.3-76.6)$ & $69.4(62.7-70.7)$ & $73.2(66.3-75.6)$ & $51.6(39.3-69.0)$ & $63.1(42.1-72.1)$ & $63.2(38.1-74.3)$ \\
\hline Protein & $0.51(0.18-0.78)^{\mathrm{b}}$ & $0.00(0.00-0.03)^{\mathrm{a}}$ & $0.30(0.05-0.71)^{\mathrm{b}}$ & $0.47(0.17-0.81)^{\mathrm{c}}$ & $0.00(0.00-0.08)^{\mathrm{a}}$ & $0.05(0.00-0.68)^{\mathrm{b}}$ \\
\hline Total Carbohydrate & $0.86(0.20-0.98)$ & $0.43(0.04-0.82)$ & $0.20(0.01-0.62)$ & $0.44(0.03-0.68)$ & $0.09(0.00-0.75)$ & $0.24(0.00-0.52)$ \\
\hline Total Fat & $0.69(0.50-1.00)$ & $0.93(0.70-0.99)$ & $1.00(0.85-1.00)$ & $1.00(0.82-1.00)$ & $0.99(0.84-1.00)$ & $1.00(0.93-1.00)$ \\
\hline PUFA & $0.44(0.25-0.80)$ & $0.35(0.07-0.73)$ & $0.14(0.02-0.60)$ & $0.49(0.21-0.76)$ & $0.49(0.07-0.83)$ & $0.66(0.24-0.91)$ \\
\hline Fibre & $0.19(0.02-0.54)^{\mathrm{c}}$ & $0.00(0.00-0.00)^{\mathrm{a}}$ & $0.00(0.00-0.00)^{\mathrm{b}}$ & $0.02(0.00-0.06)^{c}$ & $0.00(0.00-0.00)^{\mathrm{a}}$ & $0.00(0.00-0.00)^{\mathrm{b}}$ \\
\hline Vitamin A & $0.78(0.35-0.94)$ & $1.00(0.74-1.00)$ & $1.00(1.00-1.00)$ & $0.67(0.44-0.96)$ & $0.99(0.53-1.00)$ & $0.67(0.01-1.00)$ \\
\hline Thiamin & $0.85(0.48-0.98)$ & $1.00(0.90-1.00)$ & $1.00(1.00-1.00)$ & $0.61(0.34-0.81)$ & $1.00(0.40-1.00)$ & $0.97(0.05-1.00)$ \\
\hline Riboflavin & $0.96(0.81-0.98)$ & $1.00(0.91-1.00)$ & $1.00(1.00-1.00)$ & $0.83(0.57-0.93)$ & $1.00(0.65-1.00)$ & $0.97(0.41-1.00)$ \\
\hline Niacin & $0.99(0.76-1.00)$ & $1.00(0.99-1.00)$ & $1.00(1.00-1.00)$ & $0.93(0.85-0.99)$ & $1.00(0.89-1.00)$ & $1.00(0.65-1.00)$ \\
\hline Vitamin B-6 & $0.81(0.54-0.99)$ & $1.00(0.77-1.00)$ & $1.00(1.00-1.00)$ & $0.44(0.11-0.96)$ & $1.00(0.17-1.00)$ & $0.98(0.04-1.00)$ \\
\hline Folate & $0.85(0.32-0.97)$ & $0.94(0.58-1.00)$ & $0.98(0.94-1.00)$ & $0.56(0.30-0.81)$ & $0.86(0.42-0.99)$ & $0.86(0.02-1.00)$ \\
\hline Vitamin B-12 & $0.88(0.75-0.98)$ & $0.81(0.42-0.96)$ & $0.94(0.84-1.00)$ & $0.87(0.76-0.97)$ & $0.91(0.77-0.99)$ & $0.83(0.66-1.00)$ \\
\hline
\end{tabular}




\begin{tabular}{lllllll} 
Vitamin C & $0.75(0.25-0.95)$ & $1.00(0.64-1.00)$ & $1.00(1.00-1.00)$ & $0.26(0.00-0.82)^{\mathrm{a}}$ & $1.00(0.56-1.00)^{\mathrm{b}}$ & $0.94(0.06-1.00)^{\mathrm{ab}}$ \\
Vitamin D & $0.01(0.00-0.20)^{\mathrm{a}}$ & $0.99(0.50-1.00)^{\mathrm{b}}$ & $1.00(0.97-1.00)^{\mathrm{b}}$ & $0.02(0.00-0.58)^{\mathrm{a}}$ & $0.96(0.17-1.00)^{\mathrm{b}}$ & $0.71(0.31-0.99)^{\mathrm{b}}$ \\
Vitamin E & $0.34(0.11-0.94)$ & $0.97(0.46-1.00)$ & $1.00(0.95-1.00)$ & $0.18(0.02-0.44)$ & $0.95(0.03-0.99)$ & $0.71(0.17-1.00)$ \\
Calcium & $0.87(0.70-0.97)$ & $1.00(0.93-1.00)$ & $1.00(0.98-1.00)$ & $0.82(0.43-0.97)$ & $0.85(0.02-1.00)$ & $0.44(0.04-1.00)$ \\
Magnesium & $0.00(0.00-0.00)$ & $0.00(0.00-0.00)$ & $0.00(0.00-0.00)$ & $0.00(0.00-0.00)$ & $0.00(0.00-0.00)$ & $0.00(0.00-0.00)$ \\
Zinc & $0.75(0.37-0.94)$ & $1.00(0.86-1.00)$ & $1.00(1.00-1.00)$ & $0.58(0.27-0.86)$ & $1.00(0.15-1.00)$ & $0.94(0.13-1.00)$ \\
Phosphorus & $1.00(1.00-1.00)^{\mathrm{c}}$ & $0.59(0.03-0.96)^{\mathrm{a}}$ & $0.90(0.78-0.99)^{\mathrm{b}}$ & $0.99(0.96-1.00)^{\mathrm{c}}$ & $0.60(0.04-0.87)^{\mathrm{a}}$ & $0.86(0.14-1.00)^{\mathrm{b}}$ \\
Potassium & $0.69(0.45-0.93)^{\mathrm{b}}$ & $0.01(0.00-0.29)^{\mathrm{a}}$ & $0.01(0.00-0.15)^{\mathrm{a}}$ & $0.39(0.14-0.89)^{\mathrm{c}}$ & $0.00(0.00-0.02)^{\mathrm{a}}$ & $0.03(0.00-0.12)^{\mathrm{b}}$ \\
Iron & $0.93(0.85-1.00)$ & $1.00(0.85-1.00)$ & $1.00(0.96-1.00)$ & $0.93(0.55-1.00)$ & $1.00(0.45-1.00)$ & $0.85(0.15-1.00)$ \\
\hline
\end{tabular}

${ }^{1}$ Labeled medians or percentages without a common letter differ between time-points for each surgical model, as tested by paired pairwise post hoc comparisons with Holm-Bonferroni correction or paired McNemar test. 
TABLE 4. Metabolic and nutritional parameters according the surgical models at baseline, 3 months, 6 months and 12 months ${ }^{1}$

\begin{tabular}{|c|c|c|c|c|c|c|c|c|}
\hline & \multicolumn{4}{|c|}{ GBP } & \multicolumn{4}{|c|}{ SG } \\
\hline & $\begin{array}{c}\text { Baseline } \\
n=22\end{array}$ & $\begin{array}{c}3 \text { months } \\
n=22\end{array}$ & $\begin{array}{c}6 \text { months } \\
n=22\end{array}$ & $\begin{array}{c}12 \text { months } \\
n=14\end{array}$ & $\begin{array}{c}\text { Baseline } \\
\mathrm{n}=30\end{array}$ & $\begin{array}{c}3 \text { months } \\
n=30\end{array}$ & $\begin{array}{c}6 \text { months } \\
n=30\end{array}$ & $\begin{array}{c}12 \text { months } \\
n=19\end{array}$ \\
\hline Hemoglobin (g/dl) & $13.9(13.0-14.7)$ & $13.9(13.4-14.7)$ & $13.8(13.5-14.1)$ & $13.7(13.3-14.1)$ & $13.7(13.2-14.5)$ & $13.7(12.9-14.4)$ & $13.6(13.1-14.1)$ & $13.4(13.0-14.1)$ \\
\hline$<12 \mathrm{~g} / \mathrm{dl} \mathrm{N}(\%)$ & $2(9)$ & $0(0)$ & $1(5)$ & $1(7)$ & $0(0)$ & $1(3)$ & $0(0)$ & $1(5)$ \\
\hline Ferritin $(\mu \mathrm{g} / \mathrm{l})$ & $115(62-201)$ & $86(69-188)$ & $96(65-199)$ & $100(58-166)$ & $121(39-230)$ & $154(92-266)$ & $144(92-234)$ & $144(82-176)$ \\
\hline$<30 \mu \mathrm{g} / 1 \mathrm{~N}(\%)$ & $3(14)$ & $0(0)$ & $1(5)$ & $1(7)$ & $3(10)$ & $1(3)$ & $1(3)$ & $1(5)$ \\
\hline Iron $(\mu \mathrm{mol} / \mathrm{l})$ & $14.0(10.0-16.0)$ & $13.0(12.0-17.0)$ & $15.0(13.0-18.0)$ & $15.0(12.0-18.0)$ & $15.0(12.0-22.0)$ & $16.0(14.0-19.0)$ & $17.0(13.0-19.0)$ & $16.5(130-19.0)$ \\
\hline$<9 \mu \mathrm{mol} / 1 \mathrm{~N}(\%)$ & $4(18)$ & $0(0)$ & $0(0)$ & $0(0)$ & $2(7)$ & $2(7)$ & $0(0)$ & $1(5)$ \\
\hline Transferrin (g/l) & $3.1(2.7-3.1)$ & $2.3(2.2-2.8)$ & $2.4(2.1-2.8)$ & $2.5(2.0-2.8)$ & $2.7(2.5-2.9)$ & $2.4(2.2-2.7)$ & $2.5(2.3-2.7)$ & $2.6(2.3-2.7)$ \\
\hline$>3.1 \mathrm{~g} / \mathrm{l} \mathrm{N}(\%)$ & $3(14)$ & $2(9)$ & $2(9)$ & $1(7)$ & $3(10)$ & $0(0)$ & $1(3)$ & $0(0)$ \\
\hline Total iron binding capacity $(\mu \mathrm{mol} / \mathrm{l})$ & $67.5(61.0-76.0)$ & $58.0(55.0-71.0)$ & $59.0(53.0-69.0)$ & $62.0(51.0-70.0)$ & $66.5(61.0-72.0)$ & $61.0(56.0-67.0)$ & $62.0(58.0-67.0)$ & $64.0(57.0-67.0)$ \\
\hline$>80 \mu \mathrm{mol} / 1 \mathrm{~N}(\%)$ & $1(5)$ & $2(9)$ & $1(5)$ & $1(7)$ & $2(7)$ & $0(0)$ & $1(3)$ & $0(0)$ \\
\hline Transferrin saturation coefficient $(\%)$ & $0.21(0.16-0.26)$ & $0.22(0.17-0.24)$ & $0.25(0.19-0.32)$ & $0.24(0.19-0.33)$ & $0.25(0.18-0.33)$ & $0.29(0.23-0.33)$ & $0.28(0.20-0.32)$ & $0.25(0.23-0.29)$ \\
\hline$<0.15 \% \mathrm{~N}(\%)$ & $5(23)$ & $3(14)$ & $1(5)$ & $3(21)$ & $2(7)$ & $1(3)$ & $1(3)$ & $1(5)$ \\
\hline Albumin (g/l) & $35.5(33.0-37.0)^{\mathrm{a}}$ & $39.0(36.0-41.0)^{\mathrm{b}}$ & $38.0(36.0-41.0)^{\mathrm{b}}$ & $39.0(37.0-40.0)^{\mathrm{b}}$ & $37.0(35.0-39.0)^{\mathrm{a}}$ & $40.0(37.0-42.0)^{\mathrm{b}}$ & $40.0(38.0-42.0)^{\mathrm{b}}$ & $41.0(38.0-42.0)^{\mathrm{b}}$ \\
\hline$<37 \mathrm{~g} / \mathrm{l} \mathrm{N}(\%)$ & $13(59)$ & $7(32)$ & $6(27)$ & $3(21)$ & $14(47)$ & $6(20)$ & $2(7)$ & $3(16)$ \\
\hline Prealbumin (g/l) & $0.25(0.19-0.30)^{\mathrm{b}}$ & $0.20(0.16-0.21)^{\mathrm{a}}$ & $0.20(0.19-0.22)^{\mathrm{a}}$ & $0.20(0.18-0.0 .25)^{\mathrm{ab}}$ & $0.23(0.21-0.25)^{\mathrm{b}}$ & $0.18(0.17-0.21)^{\mathrm{a}}$ & $0.19(0.18-0.21)^{\mathrm{a}}$ & $0.19(0.18-0.22)^{\mathrm{a}}$ \\
\hline$<0.2 \mathrm{~g} / \mathrm{L} \mathrm{N}(\%)$ & $6(27)$ & $8(37)$ & $10(45)$ & $5(38)$ & $5(17)^{\mathrm{a}}$ & $17(57)^{\mathrm{b}}$ & $15(50)^{\mathrm{b}}$ & $10(52)^{\mathrm{b}}$ \\
\hline Calcium (mmol/l) & $2.29(2.24-2.37)$ & $2.39(2.33-2.43)$ & $2.37(2.28-2.39)$ & $2.31(2.26-2.39)$ & $2.31(2.24-2.38)$ & $2.37(2.31-2.44)$ & $2.31(2.28-2.38)$ & $2.33(2.31-2.38)$ \\
\hline 25(OH)-vitamin-D3 (ng/ml) & $13.0(10.0-23.0)^{\mathrm{a}}$ & I & $29.5(26.5-32.0)^{\mathrm{b}}$ & $27.0(22.0-29.0)^{\mathrm{b}}$ & $17.0(11.0-23.0)^{\mathrm{a}}$ & I & $26.9(22.5-30.5)^{\mathrm{b}}$ & $25.0(20.0-30.0)^{\mathrm{b}}$ \\
\hline$<30 \mathrm{ng} / \mathrm{ml} \mathrm{N}(\%)$ & $19(86)$ & I & $10(45)$ & $10(71)$ & $25(83)$ & l & $18(60)$ & $13(68)$ \\
\hline Parathyroid hormone (pg/ml) & $48.3(41.5-58.9)$ & I & I & $44.1(35.1-47.1)$ & $46.8(36.4-54.0)$ & I & I & $39.5(32.3-43.3)$ \\
\hline$>45 \mathrm{pg} / \mathrm{ml} \mathrm{N}(\%)$ & $13(59)$ & l & I & $6(43)$ & $15(50)$ & l & l & $4(21)$ \\
\hline Thiamin (nmol/l) & $157(150-174)$ & I & $193(155-193)$ & $197(174-215)$ & $147(134-175)$ & I & $177(158-191)$ & $181(149-218)$ \\
\hline$<126 \mathrm{nmol} / \mathrm{l} \mathrm{N}(\%)$ & $2(9)$ & / & $1(5)$ & $0(0)$ & $5(17)$ & I & $1(3)$ & $0(0)$ \\
\hline
\end{tabular}




\section{Erythrocyte folate (nmol/l)}

$<945 \mathrm{nmol} / \mathrm{l} \mathrm{N}(\%)$

Serum folate (nmol/l)

Vitamin B-12 (pmol/l)

$<140 \mathrm{pmol} / \mathrm{l} \mathrm{N}(\%)$

\section{7 (1023-1429) /}

4 (18)

$16.8(12.9-24.0)$

284 (209-334)

$1(5)$
1760 (1457-1961) 1940 (1421-2169)

$2(9)$

$26.9(22.8-33.4) \quad 27.9(22.8-41.0)$

$252(227-345) \quad 221(195-278)$

$0(0)$

$1234(1036-1377)^{\mathrm{a}} \quad /$

5 (17)

$17.7(14.7-20.5)^{\mathrm{a}}$

293 (248-358)
$0(0)$

$22.8(18.4-28.4)^{\mathrm{b}} \quad 20.2(15.6-26.4)^{\mathrm{b}}$

$311(224-464) \quad 311(216-432)$

$0(0)$

${ }^{1}$ Labeled medians or percentages without a common letter differ between time-points for each surgical model, as tested by paired pairwise post hoc comparisons with Holm-Bonferroni correction or paired McNemar test. Normal ranges are as follows: hemoglobin [12-17] g/dl; ferritin

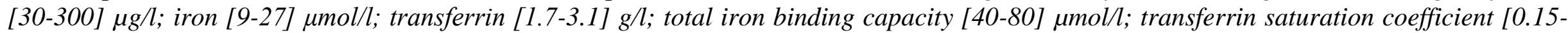
$0.35] \%$; albumin [37-50] g/l; prealbumin [0.2-0.35] g/l; calcium [2.1-2.65] mmol/l; 25(OH)-vitamin-D3 [30-100] ng/ml; thiamin [126-250] nmol/l; serum folate [7-39.5] nmol/l, vitamin B-12 [140-490] pmol/l. 


\section{FIGURE LEGENDS}

FIGURE 1. Changes in body composition in the GBP and SG groups at baseline (T0) and along the follow-up (T3, T6 and T12).

Results are expressed as means \pm SDs. Evolution of body composition during follow-up. Gastric sleeve in grey and GBP in black; top left panel fat free mass; top right panel total fat mass, low left panel appendicular fat free mass (i.e. arms + legs), low right panel appendicular fat mass. Labeled means without a common letter differ between time-points for each surgical model, as tested by paired pairwise post hoc comparisons with Holm-Bonferroni correction. No significant difference between GBP and SG was observed. 


\section{SuPPlemental Data}

SUPPLEMENTAL TABLE 1. PANDiet scores and probabilities of nutrient adequacy according to the surgical models at baseline, 3 months and 12 months (calculated from foods only) ${ }^{1}$

\begin{tabular}{|c|c|c|c|c|c|c|}
\hline & \multicolumn{3}{|c|}{ GBP } & \multicolumn{3}{|c|}{ SG } \\
\hline & $\begin{array}{c}\text { Baseline } \\
\mathrm{n}=22\end{array}$ & $\begin{array}{c}3 \text { months } \\
n=22\end{array}$ & $\begin{array}{c}12 \text { months } \\
n=14\end{array}$ & $\begin{array}{c}\text { Baseline } \\
\mathrm{n}=30\end{array}$ & $\begin{array}{c}3 \text { months } \\
n=30\end{array}$ & $\begin{array}{c}12 \text { months } \\
n=19\end{array}$ \\
\hline PANDiet & $66.0(60.7-70.5)^{\mathrm{b}}$ & $51.6(47.8-53.7)^{\mathrm{a}}$ & $52.1(46.1-57.6)^{\mathrm{a}}$ & $57.7(54.0-62.1)^{\mathrm{b}}$ & $47.6(40.9-53.0)^{\mathrm{a}}$ & $52.9(46.7-60.6)^{\mathrm{b}}$ \\
\hline Moderation Sub-score & $64.6(58.6-85.6)$ & $82.1(75.6-85.3)$ & $74.3(61.8-81.2)$ & $63.2(51.3-73.5)^{\mathrm{a}}$ & $70.9(63.8-80.0)^{\mathrm{b}}$ & $73.1(66.7-78.1)^{\mathrm{b}}$ \\
\hline Protein & $1.00(1.00-1.00)$ & $1.00(1.00-1.00)$ & $1.00(1.00-1.00)$ & $1.00(1.00-1.00)$ & $1.00(1.00-1.00)$ & $1.00(1.00-1.00)$ \\
\hline Total Carbohydrate & $1.00(1.00-1.00)$ & $1.00(0.97-1.00)$ & $1.00(0.98-1.00)$ & $1.00(1.00-1.00)$ & $1.00(1.00-1.00)$ & $1.00(1.00-1.00)$ \\
\hline Total Fat & $0.99(.035-1.00)$ & $0.81(0.58-0.93)$ & $0.59(0.05-0.95)$ & $0.84(0.52-0.96)$ & $0.42(0.02-0.84)$ & $0.57(0.05-0.89)$ \\
\hline SFA & $0.19(0.04-0.61)$ & $0.12(0.02-0.35)$ & $0.02(0.00-0.29)$ & $0.05(0.00-0.30)$ & $0.02(0.00-0.15)$ & $0.10(0.00-0.28)$ \\
\hline Cholesterol & $0.66(0.36-0.99)^{\mathrm{a}}$ & $0.99(0.94-1.00)^{\mathrm{b}}$ & $0.95(0.51-0.99)^{\mathrm{ab}}$ & $0.41(0.15-0.79)^{\mathrm{a}}$ & $0.98(0.65-1.00)^{\mathrm{b}}$ & $1.00(0.65-1.00)^{\mathrm{b}}$ \\
\hline Sodium & $0.43(0.18-0.77)^{\mathrm{a}}$ & $1.00(0.96-1.00)^{\mathrm{c}}$ & $0.97(0.71-1.00)^{\mathrm{b}}$ & $0.51(0.01-0.78)^{\mathrm{a}}$ & $1.00(0.97-1.00)^{\mathrm{b}}$ & $0.99(0.70-1.00)^{\mathrm{b}}$ \\
\hline Adequacy Sub-score & $60.8(53.0-72.0)^{\mathrm{b}}$ & $22.1(14.9-34.5)^{\mathrm{a}}$ & $30.3(22.8-42.8)^{\mathrm{a}}$ & $51.6(38.4-69.0)^{\mathrm{c}}$ & $20.6(11.9-35.2)^{\mathrm{a}}$ & $27.0(16.8-44.4)^{\mathrm{b}}$ \\
\hline Protein & $0.51(0.18-0.78)^{\mathrm{b}}$ & $0.00(0.00-0.03)^{\mathrm{a}}$ & $0.30(0.05-0.71)^{\mathrm{b}}$ & $0.47(0.17-0.81)^{\mathrm{c}}$ & $0.00(0.00-0.08)^{\mathrm{a}}$ & $0.05(0.00-0.68)^{\mathrm{b}}$ \\
\hline Total Carbohydrate & $0.86(0.20-0.98)$ & $0.43(0.04-0.82)$ & $0.20(0.01-0.62)$ & $0.44(0.03-0.68)$ & $0.09(0.00-0.75)$ & $0.24(0.00-0.52)$ \\
\hline Total Fat & $0.69(0.50-1.00)$ & $0.93(0.70-0.99)$ & $1.00(0.85-1.00)$ & $1.00(0.82-1.00)$ & $0.99(0.84-1.00)$ & $1.00(0.93-1.00)$ \\
\hline PUFA & $0.44(0.25-0.80)$ & $0.35(0.07-0.73)$ & $0.14(0.02-0.60)$ & $0.49(0.21-0.76)$ & $0.49(0.07-0.83)$ & $0.66(0.24-0.91)$ \\
\hline Fibre & $0.19(0.02-0.54)^{\mathrm{c}}$ & $0.00(0.00-0.00)^{\mathrm{a}}$ & $0.00(0.00-0.00)^{\mathrm{b}}$ & $0.02(0.00-0.06)^{\mathrm{c}}$ & $0.00(0.00-0.00)^{\mathrm{a}}$ & $0.00(0.00-0.00)^{\mathrm{b}}$ \\
\hline Vitamin A & $0.71(0.30-0.91)^{\mathrm{b}}$ & $0.12(0.00-0.51)^{\mathrm{a}}$ & $0.43(0.05-0.70)^{\mathrm{ab}}$ & $0.67(0.44-0.96)^{\mathrm{b}}$ & $0.07(0.00-0.61)^{\mathrm{a}}$ & $0.16(0.00-0.59)^{\mathrm{a}}$ \\
\hline Thiamin & $0.84(0.48-0.97)^{\mathrm{b}}$ & $0.01(0.00-0.19)^{\mathrm{a}}$ & $0.16(0.01-0.42)^{\mathrm{a}}$ & $0.56(0.31-0.77)^{\mathrm{b}}$ & $0.02(0.00-0.32)^{\mathrm{a}}$ & $0.01(0.00-0.35)^{\mathrm{a}}$ \\
\hline Riboflavin & $0.89(0.79-0.98)^{\mathrm{b}}$ & $0.06(0.01-0.67)^{\mathrm{a}}$ & $0.12(0.03-0.75)^{\mathrm{a}}$ & $0.83(0.57-0.93)^{\mathrm{b}}$ & $0.21(0.00-0.46)^{\mathrm{a}}$ & $0.08(0.00-0.70)^{\mathrm{a}}$ \\
\hline Niacin & $0.99(0.76-1.00)^{\mathrm{b}}$ & $0.09(0.00-0.60)^{\mathrm{a}}$ & $0.54(0.10-0.82)^{\mathrm{a}}$ & $0.93(0.85-0.99)^{\mathrm{b}}$ & $0.34(0.00-0.84)^{\mathrm{a}}$ & $0.68(0.41-0.98)^{\mathrm{a}}$ \\
\hline Vitamin B-6 & $0.81(0.54-0.98)^{\mathrm{b}}$ & $0.00(0.00-0.04)^{\mathrm{a}}$ & $0.00(0.00-0.28)^{\mathrm{a}}$ & $0.44(0.11-0.96)^{\mathrm{b}}$ & $0.00(0.00-0.10)^{\mathrm{a}}$ & $0.01(0.00-0.08)^{\mathrm{a}}$ \\
\hline Folate & $0.83(0.32-0.97)^{\mathrm{b}}$ & $0.04(0.01-0.17)^{\mathrm{a}}$ & $0.08(0.01-0.48)^{\mathrm{a}}$ & $0.56(0.30-0.81)^{\mathrm{b}}$ & $0.03(0.00-0.18)^{\mathrm{a}}$ & $0.04(0.01-0.16)^{\mathrm{a}}$ \\
\hline Vitamin B-12 & $0.88(0.75-0.98)^{\mathrm{b}}$ & $0.31(0.02-0.80)^{\mathrm{a}}$ & $0.72(0.38-0.90)^{\mathrm{b}}$ & $0.87(0.76-0.97)^{\mathrm{b}}$ & $0.63(0.12-0.83)^{\mathrm{a}}$ & $0.73(0.25-0.86)^{\mathrm{a}}$ \\
\hline
\end{tabular}




\begin{tabular}{llllllll} 
Vitamin C & $0.63(0.20-0.95)^{\mathrm{b}}$ & $0.05(0.00-0.57)^{\mathrm{ab}}$ & $0.09(0.00-0.47)^{\mathrm{a}}$ & $0.26(0.00-0.82)^{\mathrm{b}}$ & $0.00(0.00-0.16)^{\mathrm{a}}$ & $0.04(0.00-0.21)^{\mathrm{ab}}$ \\
Vitamin D & $0.00(0.00-0.10)^{\mathrm{b}}$ & $0.00(0.00-0.00)^{\mathrm{a}}$ & $0.00(0.00-0.02)^{\mathrm{ab}}$ & $0.02(0.00-0.30)$ & $0.00(0.00-0.08)$ & $0.15(0.00-0.44)$ \\
Vitamin E & $0.29(0.08-0.89)^{\mathrm{c}}$ & $0.00(0.00-0.00)^{\mathrm{a}}$ & $0.00(0.00-0.03)^{\mathrm{b}}$ & $0.18(0.02-0.44)^{\mathrm{b}}$ & $0.00(0.00-0.01)^{\mathrm{a}}$ & $0.13(0.00-0.39)^{\mathrm{b}}$ \\
Calcium & $0.87(0.07-0.97)^{\mathrm{b}}$ & $0.28(0.00-0.77)^{\mathrm{a}}$ & $0.49(0.07-0.86)^{\mathrm{a}}$ & $0.82(0.43-0.97)^{\mathrm{b}}$ & $0.06(0.00-0.35)^{\mathrm{a}}$ & $0.08(0.00-0.56)^{\mathrm{a}}$ \\
Magnesium & $0.00(0.00-0.00)^{\mathrm{b}}$ & $0.00(0.00-0.00)^{\mathrm{a}}$ & $0.00(0.00-0.00)^{\mathrm{a}}$ & $0.00(0.00-0.00)^{\mathrm{b}}$ & $0.00(0.00-0.00)^{\mathrm{a}}$ & $0.00(0.00-0.00)^{\mathrm{a}}$ \\
Zinc & $0.72(0.35-0.90)^{\mathrm{b}}$ & $0.01(0.00-0.14)^{\mathrm{a}}$ & $0.03(0.01-0.21)^{\mathrm{a}}$ & $0.58(0.27-0.86)^{\mathrm{c}}$ & $0.01(0.00-0.06)^{\mathrm{a}}$ & $0.11(0.00-0.51)^{\mathrm{b}}$ \\
Phosphorus & $1.00(1.00-1.00)^{\mathrm{c}}$ & $0.59(0.03-0.96)^{\mathrm{a}}$ & $0.90(0.78-0.99)^{\mathrm{b}}$ & $0.99(0.96-1.00)^{\mathrm{c}}$ & $0.60(0.04-0.87)^{\mathrm{a}}$ & $0.86(0.14-1.00)^{\mathrm{b}}$ \\
Potassium & $0.69(0.45-0.93)^{\mathrm{b}}$ & $0.01(0.00-0.29)^{\mathrm{a}}$ & $0.01(0.00-0.15)^{\mathrm{a}}$ & $0.39(0.14-0.89)^{\mathrm{c}}$ & $0.00(0.00-0.02)^{\mathrm{a}}$ & $0.03(0.00-0.12)^{\mathrm{b}}$ \\
Iron & $0.85(0.65-1.00)^{\mathrm{b}}$ & $0.04(0.00-0.55)^{\mathrm{a}}$ & $0.25(0.00-0.55)^{\mathrm{a}}$ & $0.93(0.55-1.00)^{\mathrm{b}}$ & $0.10(0.00-0.45)^{\mathrm{a}}$ & $0.15(0.00-0.85)^{\mathrm{a}}$ \\
\hline
\end{tabular}

${ }^{1}$ Labeled medians without a common letter differ between time-points for each surgical model, as tested by paired pairwise post hoc comparisons with Holm-Bonferroni correction. 


\section{Micronutrient and protein deficiencies after gastric bypass and sleeve gastrectomy: a one-year follow-up}

\section{Original Contribution}

Eric O. Verger $\mathrm{PhD}^{1,2^{*}}$, Judith Aron-Wisnewsky MD, $\mathbf{P h D}^{1,2,3^{*}}$, Maria Carlota Dao $\mathrm{PhD}^{1,2}$, Brandon D. Kayser $\mathrm{PhD}^{1,2}$, Jean-Michel Oppert MD, PhD ${ }^{1}$, Jean-Luc Bouillot MD, $\mathrm{PhD}^{4}$, Adriana Torcivia MD ${ }^{5}$, Karine Clément MD, PhD. ${ }^{1,2,3}$

*These authors contributed equally to this work.

${ }^{1}$ Institute of Cardiometabolism and Nutrition, ICAN, Assistance Publique-Hôpitaux de Paris, Pitié-Salpêtrière hospital, Nutrition department, F-75013, Paris, France. ${ }^{2}$ INSERM, UMR_S U1166, NutriOmics team, F-75013, Paris, France. ${ }^{3}$ Sorbonne Universités, UPMC University Paris 06, UMR_S 1166 I, ICAN, Nutriomics team, F-75005, Paris, France. ${ }^{4}$ Assistance Publique-Hôpitaux de Paris, Visceral surgery Department, Ambroise Paré Hospital, 92100 Boulogne-Billancourt, France. ${ }^{5}$ Assistance Publique-Hôpitaux de Paris, Visceral surgery Department, Pitié-Salpêtrière hospital, F-75013, Paris, France

Email addresses: e.verger@ican-institute.org; judith.aron-wisnewsky@psl.aphp.fr; mc.dao@ican-institute.org; b.kayser@ican-institute.org; jean-michel.oppert@psl.aphp.fr; jl.bouillot@apr.aphp.fr; adriana.torcivia@psl.aphp.fr; karine.clement@psl.aphp.fr

\section{Correspondence to:}

Dr. Judith Aron-Wisnewsky and Prof. Karine Clément

Institute of Cardiometabolism and Nutrition

Institut E3M, 83 boulevard de 1'Hôpital,

75013 Paris, France

Email: judith.aron-wisnewsky@psl.aphp.fr

Tel: 33 (0) 142177541

Running title: Malnutrition after surgery

Financial Support: This project is supported by the «Contrat de Recherche Clinique » from Health ministry (CRC Fibrota P100503 - IDRCB 2011-A00759-32, recorded on clinical trial website (NCT: NCT01655017), as well as by the European Union's Seventh Framework Programme for research, technological development and demonstration under grant agreement HEALTH-F4-2012-305312 (Metacardis) and the National Agency of Research (Investissement D'Avenir, ANR-10-IAHU-05). JAW received a grant from Institut Appert and from Nestlé research. The authors declare that they have no conflict of interest.

The authors wish to thank Ms Agathe Arlotti for the coordination of programs on bariatric surgery (BARICAN program), Ms Valentine Lemoine for her help in the follow-up of the patients and Dr Florence Marchelli who contributed to clinical and biological database constitution. 


\section{AbSTRACT}

2 Background: Roux-en-Y gastric bypass (GBP) and sleeve gastrectomy (SG) have increased

3 dramatically, potentially increasing the prevalence of nutritional deficiencies. The aim of this

4 study was to analyze the effects of food restriction during the first year after bariatric surgery

5 (BS) on nutritional parameters.

6 Methods: 22 and 30 obese patients undergoing GBP and SG were prospectively followed at 7 baseline and three, six and twelve months after $\mathrm{BS}(\mathrm{N}=14$ and $\mathrm{N}=19$ at $\mathrm{T} 12)$. We evaluated 8 food intake and nutrient adequacy (T0, T3, T12), as well as serum vitamins and minerals 9 concentration (T0, T3, T6, T12).

10 Results: At baseline, GBP and SG patients had similar clinical characteristics, food intake, 11 nutrient adequacy and serum concentration. The drastic energy and food reduction led to very 12 low probabilities of adequacy for nutrients similar in both models (T3, T12). Serum analysis 13 demonstrated a continuous decrease in prealbumin during the follow-up, indicating mild 14 protein depletion in $37 \%$ and $38 \%$ of GBP patients and $57 \%$ and $52 \%$ of SG patients, 17 prevented most nutritional deficiencies.

18 Conclusion: GBP and SG have comparable effects in terms of energy and food restriction, 19 and subsequent risk of micronutrient and protein deficiencies in the first year post BS. Such results advocate for a cautious monitoring of protein intake after GPB and SG and a 21 systematic multivitamin and mineral supplementation in the first year after SG.

23 Keywords: Bariatric surgery; Roux-en-Y Gastric Bypass; Sleeve gastrectomy; Protein 24 deficiency; Multivitamin and mineral supplementation. 


\section{INTRODUCTION}

Among the few therapeutic tools to treat morbid obesity, bariatric surgery (BS) appears to be the most effective strategy as demonstrated by its ability to obtain major and sustainable weight loss along with significant improvement of obesity related-comorbidities $[1,2]$. As a result, the number of interventions has dramatically risen worldwide, and Rouxen-Y Gastric Bypass (GBP) and Sleeve Gastrectomy (SG) represented respectively 47\% and $28 \%$ of the 340,000 BS performed in 2011 [3]. Since 2008, SG has emerged to such an extent that it has become the most common procedure in several countries, as is the case in France [3]. Although the two surgical techniques and their mechanisms of action differ, they appear to be equally safe and both induce significant weight loss post-surgery [4].

GBP includes diet restriction as well as the bypass of the proximal part of the jejunum involved in nutrient absorption whereas SG is less invasive and principally restricts the volume of the stomach [5]. Therefore SG, compared to GBP, might be viewed as less likely to exacerbate the risk of micronutrient deficiencies in obese patients who are already prone to such deficiencies before surgery [6]. Nevertheless, some studies have demonstrated a considerably higher prevalence of nutrient deficiencies after SG [7-11]. Others, comparing GBP and SG, found quite similar prevalence after both procedures [12-16]. Although study designs differed, these converging results highlight the importance of daily multivitamin and mineral supplementation after both procedures, at least in the first year for SG, in accordance with the latest US guidelines [17]. While the previously mentioned studies evaluated nutrient deficiencies using serum biomarker concentrations, only very few have evaluated food and nutrient intake after GBP and SG: Freeman et al. evaluated food intake two to four years after surgery [18], Moizé et al. and Coupaye et al. evaluated the overall macronutrient intake during one year after BS but did not quantify micronutrient intake [15,19], and Moizé et al. evaluated macronutrient and some selected mineral intake during five years after BS [14]. 
Therefore, we aimed to analyze food restriction effects on the nutritional adequacy of 4

the diet, on macro and micronutrient intake evolution as well as their consequences in terms of bioclinical evolution and micronutrient serum level during one year after both GBP and SG.

\section{MATERIAL AND METHODS}

\section{Patients}

Obese candidates for either GBP or SG according to the international bariatric surgery guidelines [20] (i.e. body mass index $(\mathrm{BMI}) \geq 40 \mathrm{~kg} / \mathrm{m}^{2}$, or $\geq 35 \mathrm{~kg} / \mathrm{m}^{2}$ with at least one severe obesity-related comorbidity) were treated in the Obesity Unit of Pitié-Salpetrière Hospital, Institute of Cardio-metabolism and Nutrition, ICAN, Paris, France. Patients determined the choice of technique, and advised by a multidisciplinary panel, from the hospital based on medical history, level of corpulence and obesity-related comorbidity. Weight stable patients were enrolled consecutively in this prospective non-randomized study from January 2012. Hotel-Dieu hospital ethics committee approved the clinical protocol (number P100503 IDRCB 2011-A00759-32) which was recorded on clinical trial website (NCT: NCT01655017). Subjects gave their written informed consent prior to the study inclusion.

Medical history and clinical evaluation were obtained at baseline and during the follow-up at three (T3), six (T6) and twelve months (T12) as described elsewhere [21]. Anthropometric parameters were estimated by whole-body fan-beam DXA scanning (Hologic Discovery W, software v12.6, 2; Hologic, Bedford, MA), as previously described [22]. Variables from DXA used in the analyses were total and appendicular fat free mass (FFM, in $\mathrm{kg}$ ), and total and appendicular fat mass (FM, in $\mathrm{kg}$ ), where appendicular FFM (or FM) was calculated as the sum of FFM (or FM) from both arms and both legs. Basal metabolic rate 
(BMR) was assessed with indirect calorimetry (Deltatrac II monitor, Datex Instrumentarium

76 Corp., Helsinki, Finland) enabling the evaluation of underreporting of dietary intake [23].

\section{Dietary data and nutrient intakes}

At baseline, T3 and T12, patients completed three consecutive web-based 24h dietary records as described elsewhere [24], including two weekdays and one day on the weekend whenever possible. All foods and beverages consumed at breakfast, lunch, dinner and snacks were recorded. Validated photographs enabled patients to estimate portion size for each reported food and beverage item [25]. Patients were also asked to indicate multivitamin and mineral supplements use, specifying the product name and amount, following the nutritional deficiency prevention treatment prescribed for every patient at our center, as described in [26]. This includes supplementation during two weeks before surgery of vitamin D (once $4 \times 100,000 \mathrm{IU})$, thiamin (250 mg/day), and vitamin B-12 (250 $\mu \mathrm{g} /$ day). Fifteen days post-GBP and SG, multivitamin and mineral supplements including Azinc "Forme et vitalité" ${ }^{\circledR}$ (two capsules per day, containing $800 \mu \mathrm{g}$ vitamin $\mathrm{A}, 1.4 \mathrm{mg}$ thiamin, $200 \mu \mathrm{g}$ folate, $1 \mu \mathrm{g}$ vitamin $\mathrm{B}$ 12, $120 \mathrm{mg}$ vitamin $\mathrm{C}, 200 \mathrm{IU}$ vitamin $\mathrm{D}, 8 \mathrm{mg}$ iron and $15 \mathrm{mg}$ zinc), iron ( $2 \times 80 \mathrm{mg} /$ day), vitamin D (800 IU/day), and calcium (1,000mg/day) were started and continued for the first year in both BS procedures. Intake of nutrients derived from food were calculated using an updated version of the French database CIQUAL 2008 [27] which included more than 3,400 different food items. Nutrient intakes from multivitamin and mineral supplements were calculated using nutrient profile based on the product name. Ingested foods were categorized into 4 main food groups when possible: (i) fruit and vegetables, (ii) starchy foods, (iii) dairy products, and (iv) meat and fish. The food groups were defined according to the French National Nutrition and Health Program [28] and expressed in servings per day based on standard serving sizes [29]. 


\section{Nutrient adequacy of the diet}

Nutrient intake adequacy for each patient was calculated using the PANDiet index [30]. Briefly, probability of adequacy for each nutrient was calculated, ranging from 0 to 1 , where 1 represents a $100 \%$ probability that the usual intake is adequate (i.e. it satisfies the requirement or is not excessive compared to a reference value). According to this definition, the probabilities of adequacy were computed to obtain the Adequacy sub-score (the higher, the better the intake satisfies the nutrient requirements) and the Moderation sub-score (the higher, the less likely the intake is excessive). The PANDiet score is taken as the mean of the Adequacy and Moderation sub-scores, and ranges from 0 to 100; the higher the score, the better the nutrient adequacy of the diet. As reference values, we used French nutritional recommendations for healthy adults or European Union values when specific recommendations were lacking.

\section{Biochemical analyses}

115 Blood samples were collected after an overnight fast to measure biochemical 116 parameters using routine techniques as described [31]. Blood count and iron metabolism 117 markers (i.e. ferritin, iron, transferrin, and saturation coefficient) were assessed using routine 118 care method (nephelemetry, ferrozine colorimetry and immunoturbidimetry respectively). 119 Prealbumin was assessed by immunoturbidimetry. Serum concentrations of 25(OH)-vitamin120 D3 and parathyroid hormone (PTH) were measured by chemiluminescent assay (310600 121 Liaison XL Diasorin and 11972103 Modular E 170 Roche, respectively), vitamin B-12 and 122 folate were assessed using immunoanalysis ECL sandwich, and thiamin and vitamin B-6 were 123 assessed using HPLC [6]. Vitamin and mineral deficiencies were defined as a result below the 124 lower normal value given by the manufacturer [32]. Secondary hyperparathyroidism was 
125 defined as an elevated PTH, above the high normal laboratory value. All measurements were

conducted at baseline, T3, T6 and T12 (except for 25(OH)-vitamin-D3, PTH, thiamin, folate and vitamin B-12 at T3, and PTH at T6).

\section{Statistical analyses}

Continuous variables are presented as median and interquartile range (IQR) and frequencies as percentages. Mann-Whitney and paired Wilcoxon rank-sum tests were, respectively, used to compare continuous variables between surgical groups and time-points. Chi-squared and McNemar tests were used to compare frequencies between surgical groups and time-points, respectively. An overall $\alpha$ level of 5\% was used for statistical tests following Holm-Bonferroni correction. These analyses were conducted on both the patients who completed T3 and T6, and on the patients who completed T3, T6 and T12. Since no significant difference was observed between two groups of patients both at baseline, and 138 during the follow-up at T3 and T6, outcomes are merged when presented on tables and 139 figures. All analyses were performed using Statistical Analysis Systems statistical software 140 package version 9.3 (SAS Institute, Cary, NC, SA).

\section{RESULTS}

\section{Clinical characteristics}

Fifty-two patients were included in this study (22 GBP and 30 SG). All of them completed the first six months follow-up of this study (T3 and T6), and 33 completed the one year follow-up (T3, T6 and T12; 14 GBP and 19 SG). Importantly, the two groups were similar at baseline regarding sex, age, corpulence and body composition (Table 1). Likewise, 148 the severity of obesity related-comorbidities was similar in the two groups, except for glucose 149 intolerance, which was significantly more prevalent in the GBP group (Table 1). 
As expected, BS induced significant weight loss in both surgical techniques, however

GBP led to a significantly greater weight loss at T6 and T12 compared to SG (Table 1). More specifically, the total and appendicular FFM (in $\mathrm{kg}$ ) significantly decreased at $\mathrm{T} 3$ and then stabilized at T6 and T12 in the two groups, while the total and appendicular FM (in $\mathrm{kg}$ ) significantly decreased along the one year follow-up in the GBP group whereas it significantly decreased until T6 and then stabilized from T6 to T12 in the SG group (Figure 1). As a result, body composition significantly improved as demonstrated by changes in the percentage of FFM and FM (Table 1). GBP induced a significant improvement of obesityrelated comorbidities (except for high blood pressure (HBP)), whereas SG only led to a significant improvement of dyslipidemia at T6 and T12 (Table 1).

\section{Food and macronutrient intakes}

At baseline, no difference was observed for energy, food or macronutrient intakes between the two groups (Table 2). The BMR values revealed that patients from both groups underreported their caloric intake by $8 \%$.

After both GBP and SG, energy intake drastically decreased at T3 and slightly increased at T12, although not reaching baseline intake levels (significant at all time points, Table 2). These changes in energy intake were explained by a significant decrease in food intake at T3 in the two surgical groups (non-significant for dairy products) and a tendency for a modest increase in food intake at T12 (significant for starchy foods in the SG group, Table 2). Total protein intake drastically and significantly decreased at $\mathrm{T} 3$ in both groups, and a majority of patients reported protein intake below the recommended value of $60 \mathrm{~g} / \mathrm{day}(85.7 \%$ after GBP and $79 \%$ after SG, Table 2). Afterwards, total protein intake slightly but significantly increased at T12, although it remained below the baseline levels (Table 2). Furthermore, $61 \%$ of the patients reported low dietary protein intake $(64 \%$ and $58 \%$ 
175 respectively for GBP and SG groups, Table 2) at T12. No significant changes in

macronutrient distribution (total fat, SFA, PUFA and total carbohydrates) were observed during the follow-up in the two groups (T3 and T12, Table 2). Energy, food and macronutrient intakes were not different between the two groups during the follow-up (T3 and T12, Table 2).

\section{Nutrient adequacy of the diet}

At baseline, neither the PANDiet scores nor the probabilities of nutrient adequacy differed between the two groups (Table 3). Low probabilities of adequacy for protein were observed in both groups compared to the French adult population [30].

After both BS, the percentage of patients taking the prescribed systematic multivitamin and mineral supplements significantly increased, from baseline to T3: 14\% versus $77 \%$ for GBP and $10 \%$ vs. $76.7 \%$ for SG, as expected from the recommendations (Table 3). This high adherence was maintained at T12 with $86 \%$ and $68 \%$ respectively for GBP and SG (Table 3). Due to the supplementation, the global nutrient adequacy of the diet did not drop and rather stabilized along the follow-up (PANDiet score and Adequacy subscore were not significantly different at all time points) and the probability of adequacy for vitamin D was improved (Table 3). Of note, when the global nutrient adequacy of the diet was calculated without taking into account the prescribed supplementation, we found that it drastically decreased at T3 and barely increased at T12 (Supplemental Table 1). However, since the prescribed supplementation neither contains protein, fiber nor phosphorus, lower probabilities of adequacy for these nutrients were observed in both groups at T3 compared to baseline (Table 3). Furthermore, although the probabilities of adequacy for these four nutrients significantly increased at T12 in both groups compared to T3 due to the slight 
increase in food intake, they remained below the baseline values (except for protein in the

GBP group, Table 3).

\section{Nutritional deficiencies}

At baseline, none of the metabolic and nutritional parameters were different between the two groups (Table 4). As expected in severe obesity, $100 \%$ and $83 \%$ of the patients from the GBP and SG groups, respectively, presented 25(OH)-vitamin-D3 deficiency as seen by serum concentrations below $30 \mathrm{ng} / \mathrm{ml}$ (Table 4) with subsequent secondary hyperparathyroidism in $50 \%$ of the subjects, showing major deficiency in this population.

After both BS, prealbumin concentration drastically and significantly decreased at T3 and further stabilized at T6 and T12 (Table 4). At T12, 38\% of GBP patients and 52\% of SG patients presented mild protein depletion as shown by prealbumin concentration below the normal range of $0.2 \mathrm{~g} / \mathrm{l}$ and $21 \%$ of GBP patients and $16 \%$ of SG patients presented risk of mild protein malnutrition as shown by albumin concentration below the normal value of 37 g/l (Table 4). Of note, two patients in the GBP group and one patient in the SG group presented both mild protein depletion and risk of mild protein malnutrition. After both BS, vitamin D supplementation enabled a significant increase in $25(\mathrm{OH})$-vitamin-D3 serum concentrations at T6, which stabilized at T12 (Table 4). However, 50\% and 21\% of GBP and SG patients, respectively, still displayed secondary hyperparathyroidism at T12 (Table 4). 218 Since all patients were prescribed multivitamin and mineral supplementation, we verified 219 whether this supplementation might induce serum concentrations of selected vitamins and minerals above the normal range at T12. In fact, there were only a few such cases in the overall cohort: one with elevated serum thiamin $(700 \mathrm{nmol} / \mathrm{l})$ and one with high serum ferritin $(740 \mu \mathrm{g} / \mathrm{l})$ in the SG group, and one with elevated vitamin B12 (580 pmol/l) in the GBP group. Importantly, all such elevations remained below toxic levels. 


\section{Discussion}

To the best of our knowledge, this is the first study to assess the relationship between food intake, nutrient adequacy of the diet and nutritional biological parameters systematically measured before, three and twelve months after GBP and SG. In this study where the patients had similar clinical characteristics at baseline (except for T2D prevalence), our main findings are: (i) protein intake significantly decreases after both GBP and SG, inducing mild protein depletion in more than a third of the patients one year after both surgical techniques; (ii) even though patients after GBP experienced greater weight loss than after SG, both types of surgery induced similar food restriction effects on the nutritional adequacy of the diet and, (iii) systematic multivitamin and mineral supplementation after SG seems to prevent these nutritional deficiencies, the same way as in GBP in the first year.

After one year, we observed that GBP led to significantly greater weight loss compared to $\mathrm{SG}$, in accordance with previous data from the literature, including a large multicenter study $[33,34]$. Nevertheless, some controversy remains. Indeed other reports show that changes in body weight were similar one year after both GBP and SG [15,19,35], although these were smaller cohorts. We evaluated the evolution of body composition and observed that, in both surgeries, total FFM decreased until three months and then stabilized, whereas total FM displayed a continuous decrease during the follow-up. Our results are consistent with previous reports showing changes in body composition following GBP [22] or SG [36] as measured by DXA. Our results are also concordant with the only study comparing these outcomes after both sleeve and by-pass [19]. In that study, the continuous weight loss during one year was due to the decrease of total FM, the total FFM being spared after four months [19]. More importantly, we observed that appendicular FFM decreased until three months and then stabilized, whereas appendicular FM continued to decrease throughout the 
follow-up period in both models. Appendicular FFM represents a better surrogate of muscle mass than total FFM [37], and this is the first time that this outcome and its evolution have been studied after SG. Interestingly, the change in appendicular FFM was similar in the two surgical procedures.

After both BS, we observed that $61 \%$ of the patients reported daily protein consumption under the recommended value of $60 \mathrm{~g} / \mathrm{day}$ at T12 (64\% for GBP and $58 \%$ for SG). Our results are consistent with those of Andreu et al. and Moizé et al. who found that respectively $37 \%$ and $46 \%$ of patients had a daily protein intake below $60 \mathrm{~g} /$ day one year after BS $[19,38]$. In accordance with those findings, we did not find any difference between GBP and SG [38]. We report a prevalence of insufficient protein intake that is nearly 2-fold higher than that reported by Moizé et al. (61\% versus 37\%), which is mostly attributable to the systematic protein supplementation prescribed by these authors to all of their patients [38]. One objective of recommending a minimal protein intake of $60 \mathrm{~g} /$ day after both GBP and SG is to mitigate post-surgical FFM loss in the first months [17]. Indeed, Moizé et al. demonstrated that patients with insufficient protein intake during the follow-up lost more FFM in both SG and GBP than patients with sufficient protein intake [19]. Because skeletal muscle is the primary site of insulin-stimulated glucose disposal during euglycemia [39], loss of FFM might contribute to the development of insulin resistance and should be avoided in order to maintain the beneficial metabolic outcomes. An important goal of future long-term follow-up studies will be to determine whether insufficient protein intake following BS might result in loss of muscular strength. Furthermore, longer-term weight stabilization (and regain) should also be assessed in link with the quantity of protein intake.

After both BS, we also observed that prealbumin concentration significantly decreased, resulting in more than a third of patients exhibiting mild protein depletion. Our results are in line with the few studies that reported changes in prealbumin concentration after 
GBP or SG. All studies found lower values at T12 after GBP compared to baseline

$[15,40,41]$. Results with SG are more heterogeneous, with reports showing both lower [42] or no change in prealbumin concentrations $[15,41]$. Of note, Moizé et al. reported that $14 \%$ of GBP and $16 \%$ of SG patients experienced abnormalities in prealbumin concentrations at T12 after BS [14]. As mentioned above, this difference may be due to the systematic prescription of protein supplement in the Hospital Clinic of Barcelona [19,38]. Adequate protein intake after BS is of utmost importance to prevent the patients from experiencing hair loss, poor wound healing and adverse effects such as infections after skin repair surgery and ultimately but rarely - protein-calorie malnutrition $[43,44]$.

Although SG merely restricts the volume of the stomach without intestinal malabsorption [5], it also leads to an accelerated gastric emptying. Subsequently faster gastrointestinal passage might promote nutrient deficiencies [45], as observed in a recent study with increased faecal excretion of fatty acids [46], resulting in a state of moderate malabsorption. Furthermore, SG decreases gastric intrinsic factor and gastric acid production, two factors involved in vitamin B-12 and iron absorption. Because most of our patients took the prescribed daily multivitamin and mineral supplements one year after both GBP and SG, few patients experienced nutritional abnormalities (except for $25(\mathrm{OH})$-vitamin-D3) and there was no difference between the two surgical groups. Our results were consistent with previous data from the literature $[14,15]$. Conversely, others reported a higher risk of vitamin B-12 and 25(OH)-vitamin-D3 deficiencies after GBP compared to SG [12]. It should be noted that in these three studies, patients undergoing GBP or SG were instructed to take multivitamin and mineral supplements on a daily basis after BS. Another point to take into account, is the risk of developing undesirably high levels of micronutrient concentrations due to the systematic supplementation as was previously reported after SG [7,8,11]. Herein, we only identified one patient with serum thiamin and another with serum ferritin above normal range. Nevertheless, 
it should be noted that the risk of excessive levels in those studies were mostly observed for vitamin A and B-6, which we did not assess. Altogether, these data highlight the importance to prescribe daily multivitamin and mineral supplements after both GBP and SG at least in the first year, but also to monitor the adherence of the patients to their supplementation.

At baseline, the higher prevalence of glucose intolerance in patients undergoing GBP reflects the process of selection for different BS techniques, where GBP is the first choice for patients with T2D or glucose intolerance since it demonstrated its superiority over SG to improve glycemic status post-surgery [35]. We also observed that neither GBP nor SG enabled a significant improvement of HBP in terms of overall prevalence. Nevertheless, both the number of patients treated and the number of treatments per patients tended to decrease after both surgeries, suggesting slight improvement of HBP in this cohort of obese patients with many comorbidities. Nevertheless our data are in accordance with previous studies, which indicated that HBP may not be the best resolved comorbidity after surgery $[47,48]$.

One of the main strengths of our study is the use of a validated web-based method of dietary assessment which allowed us to provide detailed quantification of the food and nutrient intake for each patient [24]. This method allows us to assess the use of multivitamin and mineral supplements and measure adherence of the patients to the supplementation. Although the interventions were not randomized in our study, our participants had comparable clinical characteristics at baseline (except for T2D) and were provided the same systematic supplementation regardless of the surgical procedure. The main limitation concerns the relative small number of patients, especially in the group who completed the one year follow-up. This may have prevented us from detecting changes between FFM loss and 321 low protein intake after both procedures. Future studies with longer follow-up periods and 322 larger sample size are needed to determine how poor dietary habits and nutritional 323 deficiencies correlate with weight maintenance at longer term and with the improvement or 
324 resolution of obesity related co-morbidities. We intend to follow this cohort in the second 1 2325 year of their surgery to assess their evolution in terms of nutritional risks and body 4 5326 composition.

329 consequences in terms of bioclinical evolution and micronutrient serum concentrations.

330 Altogether, our results advocate for a cautious monitoring of protein intake and a systematic

331 multivitamin and mineral supplementation after both GPB and SG - at least in the first year 332 for $\mathrm{SG}$.

334 Conflict of interest. The authors declare that they have no conflict of interest. 


\section{REFERENCES}

1. Buchwald H, Avidor Y, Braunwald E, et al. Bariatric surgery: a systematic review and meta-analysis. JAMA. 2004 Oct 13;292(14):1724-37.

2. Mingrone G, Panunzi S, De Gaetano A, et al. Bariatric surgery versus conventional medical therapy for type 2 diabetes. N Engl J Med. 2012 Apr 26;366(17):1577-85.

3. Buchwald H, Oien DM. Metabolic/bariatric surgery worldwide 2011. Obes Surg. 2013 Apr;23(4):427-36.

4. Chang S-H, Stoll CRT, Song J, et al. The effectiveness and risks of bariatric surgery: an updated systematic review and meta-analysis, 2003-2012. JAMA Surg. 2014 Mar;149(3):275-87.

5. Jacobs M, Bisland W, Gomez E, et al. Laparoscopic sleeve gastrectomy: a retrospective review of 1- and 2-year results. Surg Endosc. 2009 Aug 19;24(4):781-5.

6. Aasheim ET, Hofs $\varnothing$ D, Hjelmesaeth J, et al. Vitamin status in morbidly obese patients: a cross-sectional study. Am J Clin Nutr. 2008 Feb;87(2):362-9.

7. Aarts EO, Janssen IMC, Berends FJ. The gastric sleeve: losing weight as fast as micronutrients? Obes Surg. 2011 Feb;21(2):207-11.

8. Damms-Machado A, Friedrich A, Kramer KM, et al. Pre- and postoperative nutritional deficiencies in obese patients undergoing laparoscopic sleeve gastrectomy. Obes Surg. 2012 Jun;22(6):881-9.

9. Pech N, Meyer F, Lippert H, et al. Complications and nutrient deficiencies two years after sleeve gastrectomy. BMC Surg. 2012;12:13.

10. Saif T, Strain GW, Dakin G, et al. Evaluation of nutrient status after laparoscopic sleeve gastrectomy 1, 3, and 5 years after surgery. Surg Obes Relat Dis Off J Am Soc Bariatr Surg. 2012 Oct;8(5):542-7. 
11. Van Rutte PWJ, Aarts EO, Smulders JF, et al. Nutrient deficiencies before and after sleeve gastrectomy. Obes Surg. 2014 Oct;24(10):1639-46.

12. Gehrer S, Kern B, Peters T, et al. Fewer nutrient deficiencies after laparoscopic sleeve gastrectomy (LSG) than after laparoscopic Roux-Y-gastric bypass (LRYGB)-a prospective study. Obes Surg. 2010 Apr;20(4):447-53.

13. Kehagias I, Karamanakos SN, Argentou M, et al. Randomized clinical trial of laparoscopic Roux-en-Y gastric bypass versus laparoscopic sleeve gastrectomy for the management of patients with BMI < $50 \mathrm{~kg} / \mathrm{m} 2$. Obes Surg. $2011 \mathrm{Nov} ; 21(11): 1650-6$.

14. Moizé V, Andreu A, Flores L, et al. Long-term dietary intake and nutritional deficiencies following sleeve gastrectomy or Roux-En-Y gastric bypass in a mediterranean population. J Acad Nutr Diet. 2013 Mar;113(3):400-10.

15. Coupaye M, Rivière $\mathrm{P}$, Breuil MC, et al. Comparison of nutritional status during the first year after sleeve gastrectomy and Roux-en-Y gastric bypass. Obes Surg. 2014 Feb;24(2):276-83.

16. Kwon Y, Kim HJ, Lo Menzo E, et al. Anemia, iron and vitamin B12 deficiencies after sleeve gastrectomy compared to Roux-en-Y gastric bypass: a meta-analysis. Surg Obes Relat Dis Off J Am Soc Bariatr Surg. 2014 Aug;10(4):589-97.

17. Mechanick JI, Youdim A, Jones DB, et al. Clinical Practice Guidelines for the Perioperative Nutritional, Metabolic, and Nonsurgical Support of the Bariatric Surgery Patient - 2013 Update: Cosponsored by American Association of Clinical Endocrinologists, The Obesity Society, and American Society for Metabolic \& Bariatric Surgery. Obes Silver Spring Md. 2013 Mar;21(0 1):S1-27.

18. Freeman RA, Overs SE, Zarshenas N, et al. Food tolerance and diet quality following adjustable gastric banding, sleeve gastrectomy and Roux-en-Y gastric bypass. Obes Res Clin Pract. 2014 Apr;8(2):e115-200. 
19. Moizé V, Andreu A, Rodríguez L, et al. Protein intake and lean tissue mass retention following bariatric surgery. Clin Nutr Edinb Scotl. 2013 Aug;32(4):550-5.

20. Fried M, Yumuk V, Oppert J-M, et al. Interdisciplinary European Guidelines on metabolic and bariatric surgery. Obes Facts. 2013;6(5):449-68.

21. Abdennour M, Reggio S, Le Naour G, et al. Association of adipose tissue and liver fibrosis with tissue stiffness in morbid obesity: links with diabetes and BMI loss after gastric bypass. J Clin Endocrinol Metab. 2014 Mar;99(3):898-907.

22. Ciangura C, Bouillot J-L, Lloret-Linares C, et al. Dynamics of change in total and regional body composition after gastric bypass in obese patients. Obes Silver Spring Md. 2010 Apr;18(4):760-5.

23. Nielsen BM, Nielsen MM, Toubro S, et al. Past and current body size affect validity of reported energy intake among middle-aged Danish men. J Nutr. 2009 Dec;139(12):233743.

24. Touvier M, Kesse-Guyot E, Méjean C, et al. Comparison between an interactive webbased self-administered $24 \mathrm{~h}$ dietary record and an interview by a dietitian for large-scale epidemiological studies. Br J Nutr. 2011 Apr;105(7):1055-64.

25. Le Moullec N, Deheeger M, Preziosi P, et al. Validation du manuel-photos utilisé pour l'enquête alimentaire de l'étude SU.VI.MAX. Cah Nutr Diététique. 31(3):158-64.

26. Gesquiere I, Aron-Wisnewsky J, Foulon V, et al. Medication cost is significantly reduced after Roux-en-Y gastric bypass in obese patients. Obes Surg. 2014 Nov;24(11):1896903.

27. Afssa - Table de composition nutritionnelle des aliments CIQUAL 2008 (French Food Composition Table - CIQUAL 2008). Available from:

https://pro.anses.fr/tableciqual/index.htm. 
28. Hercberg S, Chat-Yung S, Chaulia M. The French National Nutrition and Health Program: 2001-2006-2010. Int J Public Health. 2008;53(2):68-77.

29. Estaquio C, Kesse-Guyot E, Deschamps V, et al. Adherence to the French Programme National Nutrition Santé Guideline Score is associated with better nutrient intake and nutritional status. J Am Diet Assoc. 2009 Jun;109(6):1031-41.

30. Verger EO, Mariotti F, Holmes BA, et al. Evaluation of a diet quality index based on the probability of adequate nutrient intake (PANDiet) using national French and US dietary surveys. PloS One. 2012;7(8):e42155.

31. Aron-Wisnewsky J, Minville C, Tordjman J, et al. Chronic intermittent hypoxia is a major trigger for non-alcoholic fatty liver disease in morbid obese. J Hepatol. 2012 Jan;56(1):225-33.

32. Ledoux S, Msika S, Moussa F, et al. Comparison of nutritional consequences of conventional therapy of obesity, adjustable gastric banding, and gastric bypass. Obes Surg. 2006 Aug;16(8):1041-9.

33. Hutter MM, Schirmer BD, Jones DB, et al. First report from the American College of Surgeons Bariatric Surgery Center Network: laparoscopic sleeve gastrectomy has morbidity and effectiveness positioned between the band and the bypass. Ann Surg. 2011 Sep;254(3):410-420; discussion 420-422.

34. Lim DM, Taller J, Bertucci W, et al. Comparison of laparoscopic sleeve gastrectomy to laparoscopic Roux-en-Y gastric bypass for morbid obesity in a military institution. Surg Obes Relat Dis Off J Am Soc Bariatr Surg. 2014 Apr;10(2):269-76.

35. Kashyap SR, Bhatt DL, Wolski K, et al. Metabolic effects of bariatric surgery in patients with moderate obesity and type 2 diabetes: analysis of a randomized control trial comparing surgery with intensive medical treatment. Diabetes Care. 2013 Aug;36(8):2175-82. 
36. Bužga M, Zavadilová V, Holéczy $\mathrm{P}$, et al. Dietary intake and ghrelin and leptin changes after sleeve gastrectomy. Videosurgery Miniinvasive Tech. 2014 Dec;9(4):554-61.

37. Kim J, Wang Z, Heymsfield SB, et al. Total-body skeletal muscle mass: estimation by a new dual-energy X-ray absorptiometry method. Am J Clin Nutr. 2002 Aug 1;76(2):37883.

38. Andreu A, Moizé V, Rodríguez L, et al. Protein intake, body composition, and protein status following bariatric surgery. Obes Surg. 2010 Nov;20(11):1509-15.

39. DeFronzo RA, Jacot E, Jequier E, et al. The effect of insulin on the disposal of intravenous glucose. Results from indirect calorimetry and hepatic and femoral venous catheterization. Diabetes. 1981 Dec;30(12):1000-7.

40. Coupaye M, Puchaux K, Bogard C, et al. Nutritional consequences of adjustable gastric banding and gastric bypass: a 1-year prospective study. Obes Surg. 2009 Jan;19(1):5665.

41. Sallé A, Demarsy D, Poirier AL, et al. Zinc deficiency: a frequent and underestimated complication after bariatric surgery. Obes Surg. 2010 Dec;20(12):1660-70.

42. Friedrich AE, Damms-Machado A, Meile T, et al. Laparoscopic sleeve gastrectomy compared to a multidisciplinary weight loss program for obesity--effects on body composition and protein status. Obes Surg. 2013 Dec;23(12):1957-65.

43. Agha-Mohammadi S, Hurwitz DJ. Nutritional deficiency of post-bariatric surgery body contouring patients: what every plastic surgeon should know. Plast Reconstr Surg. 2008 Aug;122(2):604-13.

44. Faria SL, Faria OP, Buffington C, de Almeida Cardeal M, Ito MK. Dietary protein intake and bariatric surgery patients: a review. Obes Surg. 2011 Nov;21(11):1798-805.

45. Melissas J, Daskalakis M, Koukouraki S, et al. Sleeve Gastrectomy - A "Food Limiting" Operation. Obes Surg. 2008 Jul 29;18(10):1251-6. 
46. Damms-Machado A, Mitra S, Schollenberger AE, et al. Effects of Surgical and Dietary Weight Loss Therapy for Obesity on Gut Microbiota Composition and Nutrient Absorption. BioMed Res Int. 2015 Feb 1;2015:e806248.

47. Sjöström L, Narbro K, Sjöström CD, et al. Effects of bariatric surgery on mortality in Swedish obese subjects. Engl J Med. 2007 Aug 23;357(8):741-52.

48. Gesquiere I, Aron-Wisnewsky J, Foulon V, et al. Medication cost is significantly reduced after Roux-en-Y gastric bypass in obese patients. Obes Surg. 2014 Nov;24(11):1896-903. 


\section{TABLES}

TABLE 1. Anthropometric parameters and clinical characteristics according the surgical models at baseline, 3 months, 6 months and 12 months ${ }^{1}$.

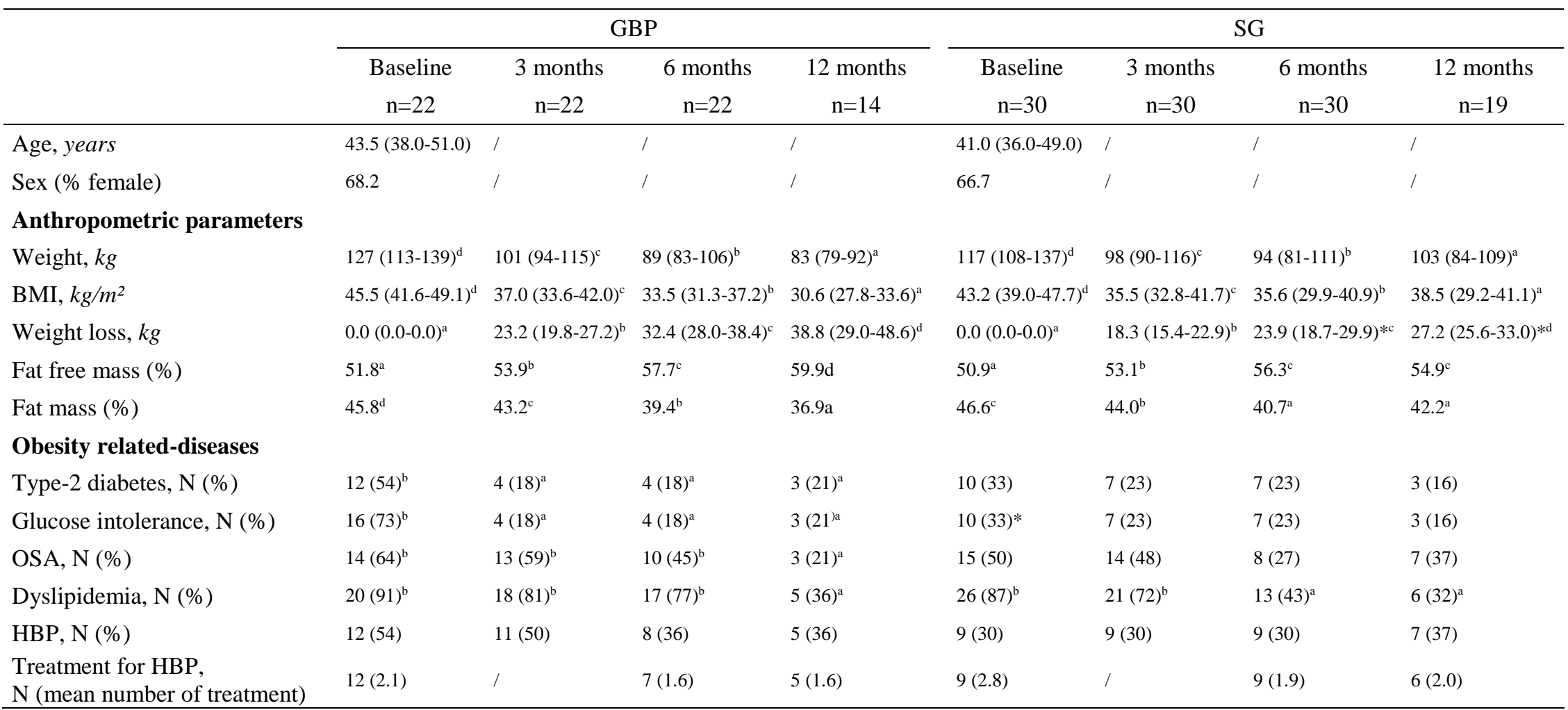

${ }^{1}$ Labeled medians or percentages without a common letter differ between time-points for each surgical model, as tested by paired pairwise post hoc comparisons with Holm-Bonferroni correction or paired McNemar test. *Represents significant differences between GBP and SG. Glucose intolerance is defined as either fasting hyperglycemia $(1 \mathrm{~g} / \mathrm{l} \leq G<1.26 \mathrm{~g} / \mathrm{l})$ or $6 \% \leq H B A 1 \mathrm{c}<6.5 \%)$; dyslipidemia is defined as a patient with treatment (statin or fibrate) or hypertriglyceridemia $\geq 1.5 \mathrm{~g} / \mathrm{l}$ or hypoHDL $<0.5 \mathrm{~g} / \mathrm{l}$ for women and hypoHDL $<0.4 \mathrm{~g} / \mathrm{l}$ for men; High blood pressure $(\mathrm{HBP})$ is defined as a systolic blood pressure $>140 \mathrm{mmHg}$ and/or a diastolic blood pressure >90mmHg or patients with an anti-hypertensive treatment; obstructive sleep apnea (OSA) is defined as an Index Apnea Hypopnea >5/hour with or without treatment.) 
TABLE 2. Energy, food and macronutrient intakes according to the surgical models at baseline, 3 months and 12 months $^{1}$.

\begin{tabular}{|c|c|c|c|c|c|c|}
\hline & \multicolumn{3}{|c|}{ GBP } & \multicolumn{3}{|c|}{ SG } \\
\hline & $\begin{array}{c}\text { Baseline } \\
n=22\end{array}$ & $\begin{array}{c}3 \text { months } \\
n=22\end{array}$ & $\begin{array}{c}12 \text { months } \\
n=14\end{array}$ & $\begin{array}{c}\text { Baseline } \\
n=30\end{array}$ & $\begin{array}{c}3 \text { months } \\
n=30\end{array}$ & $\begin{array}{c}12 \text { months } \\
\mathrm{n}=19\end{array}$ \\
\hline \multicolumn{7}{|l|}{ Energy and food intakes } \\
\hline Energy intake, $\mathrm{kcal} / \mathrm{d}$ & $2005(1539-2266)^{\mathrm{c}}$ & $711(615-1006)^{\mathrm{a}}$ & $1226(8133-1559)^{\mathrm{b}}$ & $1658(1445-2395)^{\mathrm{c}}$ & $833(539-1108)^{\mathrm{a}}$ & $1078(793-1354)^{b}$ \\
\hline $\mathrm{BMR}, \mathrm{kcal} / \mathrm{d}$ & $2179(2005-2409)^{\mathrm{c}}$ & $1770(1702-2072)^{b}$ & $1653(1480-1791)^{\mathrm{a}}$ & $1959(1853-2218)^{\mathrm{c}}$ & $1742(1593-1894)^{b}$ & $1686(1565-1963)^{\mathrm{a}}$ \\
\hline Fruit and vegetables, serving/d & $4.8(3.2-7.0)^{\mathrm{b}}$ & $2.2(0.8-3.2)^{\mathrm{a}}$ & $2.1(1.5-3.9)^{\mathrm{ab}}$ & $3.0(1.6-4.3)^{\mathrm{b}}$ & $1.5(0.8-2.1)^{\mathrm{a}}$ & $1.4(1.0-2.6)^{\mathrm{ab}}$ \\
\hline Starchy foods, serving/d & $2.8(2.1-3.7)^{\mathrm{b}}$ & $0.7(0.3-1.2)^{\mathrm{a}}$ & $1.1(0.8-1.6)^{\mathrm{a}}$ & $2.6(2.1-3.3)^{\mathrm{c}}$ & $0.7(0.3-1.1)^{\mathrm{a}}$ & $1.2(0.7-1.7)^{\mathrm{b}}$ \\
\hline Dairy products, serving/d & $2.1(1.3-3.1)$ & $1.7(0.5-2.6)$ & $2.1(0.8-2.5)$ & $1.6(1.0-2.4)$ & $1.4(0.6-1.9)$ & $1.2(0.7-1.7)$ \\
\hline Meat and fish, serving/d & $1.4(1.0-2.6)^{\mathrm{b}}$ & $0.8(0.6-1.1)^{\mathrm{a}}$ & $0.7(0.4-1.6)^{\mathrm{ab}}$ & $1.6(1.1-2.5)^{\mathrm{b}}$ & $0.9(0.6-1.4)^{\mathrm{a}}$ & $1.0(0.7-1.8)^{\mathrm{ab}}$ \\
\hline \multicolumn{7}{|l|}{ Macronutrient intakes } \\
\hline Protein, $g / d$ & $83.5(70.6-105.6)^{\mathrm{c}}$ & $41,7(24,0-49,0)^{\mathrm{a}}$ & $50,4(36,9-65,2)^{\mathrm{b}}$ & $78,3(64.0-107,2)^{\mathrm{c}}$ & $41,2(26,8-52,6)^{\mathrm{a}}$ & $51,8(36,4-65,3)^{\mathrm{b}}$ \\
\hline $\mathrm{N}(\%)<60 \mathrm{~g} / d$ & $2(9)^{\mathrm{a}}$ & $19(86)^{b}$ & $9(64)^{\mathrm{b}}$ & $4(13)^{\mathrm{a}}$ & $26(87)^{b}$ & $11(58)^{\mathrm{b}}$ \\
\hline Protein, $g / \mathrm{kg} / \mathrm{d}$ & $0.66(0.57-0.73)^{\mathrm{b}}$ & $0,38(0,24-0,46)^{\mathrm{a}}$ & $0,59(0,48-0,715)^{\mathrm{b}}$ & $0,65(0,57-0,80)^{\mathrm{c}}$ & $0,39(0,29-0,50)^{\mathrm{a}}$ & $0,46(0,39-0,74)^{\mathrm{b}}$ \\
\hline Total Lipid, \%EI/d & $32.0(30.0-40.6)$ & $36,8(32,4-39,3)$ & $38,8(33,6-45,6)$ & $37,4(33,2-39,9)$ & $41,6(35,8-44,7)$ & $39,5(37,1-44,5)$ \\
\hline $\mathrm{SFA}, \% E I / d$ & $14.7(11.3-16.4)$ & $15,5(13,1-16,6)$ & $17,4(13,7-20,9)$ & $15,6(14,5-18,7)$ & $17,4(15,3-19,6)$ & $15,8(13,7-19,4)$ \\
\hline PUFA, $\% E I / d$ & $4.8(4.2-5.8)$ & $4,3(3,2-6,4)$ & $3,5(3,0-5,5)$ & $5.0(4,0-5,9)$ & $5,0(3,3-6,4)$ & $5,6(4,3-8.0)$ \\
\hline Total Carbohydrate, $\% E I / d$ & $47.8(42.0-49.7)$ & $44.0(38.9-49.2)$ & $42,2(35,4-47,1)$ & $44.1(40.0-46.7)$ & $37.4(32.3-46.8)$ & $42,4(33,4-45,1)$ \\
\hline
\end{tabular}

${ }^{1}$ Labeled medians or percentages without a common letter differ between time-points for each surgical model, as tested by paired pairwise post hoc comparisons with Holm-Bonferroni correction or paired McNemar test. 
TABLE 3. Multivitamin and mineral supplementation, PANDiet scores and probabilities of nutrient adequacy according to the surgical models at baseline, 3 months and 12 months ${ }^{1}$

\begin{tabular}{|c|c|c|c|c|c|c|}
\hline & \multicolumn{3}{|c|}{ GBP } & \multicolumn{3}{|c|}{ SG } \\
\hline & $\begin{array}{c}\text { Baseline } \\
n=22\end{array}$ & $\begin{array}{c}3 \text { months } \\
n=22\end{array}$ & $\begin{array}{c}12 \text { months } \\
n=14\end{array}$ & $\begin{array}{c}\text { Baseline } \\
n=30\end{array}$ & $\begin{array}{c}3 \text { months } \\
n=30\end{array}$ & $\begin{array}{c}12 \text { months } \\
n=19\end{array}$ \\
\hline Supplementation, N (\%) & $3(14)^{\mathrm{a}}$ & $17(77)^{b}$ & $12(86)^{b}$ & $3(10)^{\mathrm{a}}$ & $23(77)^{b}$ & $13(68)^{b}$ \\
\hline PANDiet & $67.4(60.7-70.7)$ & $74.7(61.5-76.3)$ & $71.0(65.3-75.0)$ & $57.7(54.0-63.1)$ & $65.3(57.2-71.3)$ & $65.0(57.4-73.0)$ \\
\hline Moderation Sub-score & $64.6(58.6-85.6)$ & $82.1(75.6-85.3)$ & $74.3(61.8-81.2)$ & $63.2(51.3-73.5)^{\mathrm{a}}$ & $70.9(63.83-80.0)^{\mathrm{b}}$ & $73.1(66.7-78.1)^{\mathrm{b}}$ \\
\hline Protein & $1.00(1.00-1.00)$ & $1.00(1.00-1.00)$ & $1.00(1.00-1.00)$ & $1.00(1.00-1.00)$ & $1.00(1.00-1.00)$ & $1.00(1.00-1.00)$ \\
\hline Total Carbohydrate & $1.00(1.00-1.00)$ & $1.00(0.97-1.00)$ & $1.00(0.98-1.00)$ & $1.00(1.00-1.00)$ & $1.00(1.00-1.00)$ & $1.00(1.00-1.00)$ \\
\hline Total Fat & $0.99(.035-1.00)$ & $0.81(0.58-0.93)$ & $0.59(0.05-0.95)$ & $0.84(0.52-0.96)$ & $0.42(0.02-0.84)$ & $0.57(0.05-0.89)$ \\
\hline SFA & $0.19(0.04-0.61)$ & $0.12(0.02-0.35)$ & $0.02(0.00-0.29)$ & $0.05(0.00-0.30)$ & $0.02(0.00-0.15)$ & $0.10(0.00-0.28)$ \\
\hline Cholesterol & $0.66(0.36-0.99)^{\mathrm{a}}$ & $0.99(0.94-1.00)^{\mathrm{b}}$ & $0.95(0.51-0.99)^{\mathrm{ab}}$ & $0.41(0.15-0.79)^{\mathrm{a}}$ & $0.98(0.65-1.00)^{\mathrm{b}}$ & $1.00(0.65-1.00)^{\mathrm{b}}$ \\
\hline Sodium & $0.43(0.18-0.77)^{\mathrm{a}}$ & $1.00(0.96-1.00)^{\mathrm{c}}$ & $0.97(0.71-1.00)^{\mathrm{b}}$ & $0.51(0.01-0.78)^{\mathrm{a}}$ & $1.00(0.97-1.00)^{\mathrm{b}}$ & $0.99(0.70-1.00)^{\mathrm{b}}$ \\
\hline Adequacy Sub-score & $63.7(53.3-76.6)$ & $69.4(62.7-70.7)$ & $73.2(66.3-75.6)$ & $51.6(39.3-69.0)$ & $63.1(42.1-72.1)$ & $63.2(38.1-74.3)$ \\
\hline Protein & $0.51(0.18-0.78)^{\mathrm{b}}$ & $0.00(0.00-0.03)^{\mathrm{a}}$ & $0.30(0.05-0.71)^{\mathrm{b}}$ & $0.47(0.17-0.81)^{\mathrm{c}}$ & $0.00(0.00-0.08)^{\mathrm{a}}$ & $0.05(0.00-0.68)^{\mathrm{b}}$ \\
\hline Total Carbohydrate & $0.86(0.20-0.98)$ & $0.43(0.04-0.82)$ & $0.20(0.01-0.62)$ & $0.44(0.03-0.68)$ & $0.09(0.00-0.75)$ & $0.24(0.00-0.52)$ \\
\hline Total Fat & $0.69(0.50-1.00)$ & $0.93(0.70-0.99)$ & $1.00(0.85-1.00)$ & $1.00(0.82-1.00)$ & $0.99(0.84-1.00)$ & $1.00(0.93-1.00)$ \\
\hline PUFA & $0.44(0.25-0.80)$ & $0.35(0.07-0.73)$ & $0.14(0.02-0.60)$ & $0.49(0.21-0.76)$ & $0.49(0.07-0.83)$ & $0.66(0.24-0.91)$ \\
\hline Fibre & $0.19(0.02-0.54)^{\mathrm{c}}$ & $0.00(0.00-0.00)^{\mathrm{a}}$ & $0.00(0.00-0.00)^{\mathrm{b}}$ & $0.02(0.00-0.06)^{c}$ & $0.00(0.00-0.00)^{\mathrm{a}}$ & $0.00(0.00-0.00)^{\mathrm{b}}$ \\
\hline Vitamin A & $0.78(0.35-0.94)$ & $1.00(0.74-1.00)$ & $1.00(1.00-1.00)$ & $0.67(0.44-0.96)$ & $0.99(0.53-1.00)$ & $0.67(0.01-1.00)$ \\
\hline Thiamin & $0.85(0.48-0.98)$ & $1.00(0.90-1.00)$ & $1.00(1.00-1.00)$ & $0.61(0.34-0.81)$ & $1.00(0.40-1.00)$ & $0.97(0.05-1.00)$ \\
\hline Riboflavin & $0.96(0.81-0.98)$ & $1.00(0.91-1.00)$ & $1.00(1.00-1.00)$ & $0.83(0.57-0.93)$ & $1.00(0.65-1.00)$ & $0.97(0.41-1.00)$ \\
\hline Niacin & $0.99(0.76-1.00)$ & $1.00(0.99-1.00)$ & $1.00(1.00-1.00)$ & $0.93(0.85-0.99)$ & $1.00(0.89-1.00)$ & $1.00(0.65-1.00)$ \\
\hline Vitamin B-6 & $0.81(0.54-0.99)$ & $1.00(0.77-1.00)$ & $1.00(1.00-1.00)$ & $0.44(0.11-0.96)$ & $1.00(0.17-1.00)$ & $0.98(0.04-1.00)$ \\
\hline Folate & $0.85(0.32-0.97)$ & $0.94(0.58-1.00)$ & $0.98(0.94-1.00)$ & $0.56(0.30-0.81)$ & $0.86(0.42-0.99)$ & $0.86(0.02-1.00)$ \\
\hline Vitamin B-12 & $0.88(0.75-0.98)$ & $0.81(0.42-0.96)$ & $0.94(0.84-1.00)$ & $0.87(0.76-0.97)$ & $0.91(0.77-0.99)$ & $0.83(0.66-1.00)$ \\
\hline
\end{tabular}




\begin{tabular}{lllllll} 
Vitamin C & $0.75(0.25-0.95)$ & $1.00(0.64-1.00)$ & $1.00(1.00-1.00)$ & $0.26(0.00-0.82)^{\mathrm{a}}$ & $1.00(0.56-1.00)^{\mathrm{b}}$ & $0.94(0.06-1.00)^{\mathrm{ab}}$ \\
Vitamin D & $0.01(0.00-0.20)^{\mathrm{a}}$ & $0.99(0.50-1.00)^{\mathrm{b}}$ & $1.00(0.97-1.00)^{\mathrm{b}}$ & $0.02(0.00-0.58)^{\mathrm{a}}$ & $0.96(0.17-1.00)^{\mathrm{b}}$ & $0.71(0.31-0.99)^{\mathrm{b}}$ \\
Vitamin E & $0.34(0.11-0.94)$ & $0.97(0.46-1.00)$ & $1.00(0.95-1.00)$ & $0.18(0.02-0.44)$ & $0.95(0.03-0.99)$ & $0.71(0.17-1.00)$ \\
Calcium & $0.87(0.70-0.97)$ & $1.00(0.93-1.00)$ & $1.00(0.98-1.00)$ & $0.82(0.43-0.97)$ & $0.85(0.02-1.00)$ & $0.44(0.04-1.00)$ \\
Magnesium & $0.00(0.00-0.00)$ & $0.00(0.00-0.00)$ & $0.00(0.00-0.00)$ & $0.00(0.00-0.00)$ & $0.00(0.00-0.00)$ & $0.00(0.00-0.00)$ \\
Zinc & $0.75(0.37-0.94)$ & $1.00(0.86-1.00)$ & $1.00(1.00-1.00)$ & $0.58(0.27-0.86)$ & $1.00(0.15-1.00)$ & $0.94(0.13-1.00)$ \\
Phosphorus & $1.00(1.00-1.00)^{\mathrm{c}}$ & $0.59(0.03-0.96)^{\mathrm{a}}$ & $0.90(0.78-0.99)^{\mathrm{b}}$ & $0.99(0.96-1.00)^{\mathrm{c}}$ & $0.60(0.04-0.87)^{\mathrm{a}}$ & $0.86(0.14-1.00)^{\mathrm{b}}$ \\
Potassium & $0.69(0.45-0.93)^{\mathrm{b}}$ & $0.01(0.00-0.29)^{\mathrm{a}}$ & $0.01(0.00-0.15)^{\mathrm{a}}$ & $0.39(0.14-0.89)^{\mathrm{c}}$ & $0.00(0.00-0.02)^{\mathrm{a}}$ & $0.03(0.00-0.12)^{\mathrm{b}}$ \\
Iron & $0.93(0.85-1.00)$ & $1.00(0.85-1.00)$ & $1.00(0.96-1.00)$ & $0.93(0.55-1.00)$ & $1.00(0.45-1.00)$ & $0.85(0.15-1.00)$ \\
\hline
\end{tabular}

${ }^{1}$ Labeled medians or percentages without a common letter differ between time-points for each surgical model, as tested by paired pairwise post hoc comparisons with Holm-Bonferroni correction or paired McNemar test. 
TABLE 4. Metabolic and nutritional parameters according the surgical models at baseline, 3 months, 6 months and 12 months ${ }^{1}$

\begin{tabular}{|c|c|c|c|c|c|c|c|c|}
\hline & \multicolumn{4}{|c|}{ GBP } & \multicolumn{4}{|c|}{ SG } \\
\hline & $\begin{array}{c}\text { Baseline } \\
n=22\end{array}$ & $\begin{array}{c}3 \text { months } \\
n=22\end{array}$ & $\begin{array}{c}6 \text { months } \\
n=22\end{array}$ & $\begin{array}{c}12 \text { months } \\
n=14\end{array}$ & $\begin{array}{c}\text { Baseline } \\
\mathrm{n}=30\end{array}$ & $\begin{array}{c}3 \text { months } \\
n=30\end{array}$ & $\begin{array}{c}6 \text { months } \\
n=30\end{array}$ & $\begin{array}{c}12 \text { months } \\
n=19\end{array}$ \\
\hline Hemoglobin (g/dl) & $13.9(13.0-14.7)$ & $13.9(13.4-14.7)$ & $13.8(13.5-14.1)$ & $13.7(13.3-14.1)$ & $13.7(13.2-14.5)$ & $13.7(12.9-14.4)$ & $13.6(13.1-14.1)$ & $13.4(13.0-14.1)$ \\
\hline$<12 \mathrm{~g} / \mathrm{dl} \mathrm{N}(\%)$ & $2(9)$ & $0(0)$ & $1(5)$ & $1(7)$ & $0(0)$ & $1(3)$ & $0(0)$ & $1(5)$ \\
\hline Ferritin $(\mu \mathrm{g} / \mathrm{l})$ & $115(62-201)$ & $86(69-188)$ & $96(65-199)$ & $100(58-166)$ & $121(39-230)$ & $154(92-266)$ & $144(92-234)$ & $144(82-176)$ \\
\hline$<30 \mu \mathrm{g} / 1 \mathrm{~N}(\%)$ & $3(14)$ & $0(0)$ & $1(5)$ & $1(7)$ & $3(10)$ & $1(3)$ & $1(3)$ & $1(5)$ \\
\hline Iron $(\mu \mathrm{mol} / \mathrm{l})$ & $14.0(10.0-16.0)$ & $13.0(12.0-17.0)$ & $15.0(13.0-18.0)$ & $15.0(12.0-18.0)$ & $15.0(12.0-22.0)$ & $16.0(14.0-19.0)$ & $17.0(13.0-19.0)$ & $16.5(130-19.0)$ \\
\hline$<9 \mu \mathrm{mol} / 1 \mathrm{~N}(\%)$ & $4(18)$ & $0(0)$ & $0(0)$ & $0(0)$ & $2(7)$ & $2(7)$ & $0(0)$ & $1(5)$ \\
\hline Transferrin (g/l) & $3.1(2.7-3.1)$ & $2.3(2.2-2.8)$ & $2.4(2.1-2.8)$ & $2.5(2.0-2.8)$ & $2.7(2.5-2.9)$ & $2.4(2.2-2.7)$ & $2.5(2.3-2.7)$ & $2.6(2.3-2.7)$ \\
\hline$>3.1 \mathrm{~g} / \mathrm{l} \mathrm{N}(\%)$ & $3(14)$ & $2(9)$ & $2(9)$ & $1(7)$ & $3(10)$ & $0(0)$ & $1(3)$ & $0(0)$ \\
\hline Total iron binding capacity $(\mu \mathrm{mol} / \mathrm{l})$ & $67.5(61.0-76.0)$ & $58.0(55.0-71.0)$ & $59.0(53.0-69.0)$ & $62.0(51.0-70.0)$ & $66.5(61.0-72.0)$ & $61.0(56.0-67.0)$ & $62.0(58.0-67.0)$ & $64.0(57.0-67.0)$ \\
\hline$>80 \mu \mathrm{mol} / 1 \mathrm{~N}(\%)$ & $1(5)$ & $2(9)$ & $1(5)$ & $1(7)$ & $2(7)$ & $0(0)$ & $1(3)$ & $0(0)$ \\
\hline Transferrin saturation coefficient $(\%)$ & $0.21(0.16-0.26)$ & $0.22(0.17-0.24)$ & $0.25(0.19-0.32)$ & $0.24(0.19-0.33)$ & $0.25(0.18-0.33)$ & $0.29(0.23-0.33)$ & $0.28(0.20-0.32)$ & $0.25(0.23-0.29)$ \\
\hline$<0.15 \% \mathrm{~N}(\%)$ & $5(23)$ & $3(14)$ & $1(5)$ & $3(21)$ & $2(7)$ & $1(3)$ & $1(3)$ & $1(5)$ \\
\hline Albumin (g/l) & $35.5(33.0-37.0)^{\mathrm{a}}$ & $39.0(36.0-41.0)^{\mathrm{b}}$ & $38.0(36.0-41.0)^{\mathrm{b}}$ & $39.0(37.0-40.0)^{\mathrm{b}}$ & $37.0(35.0-39.0)^{\mathrm{a}}$ & $40.0(37.0-42.0)^{\mathrm{b}}$ & $40.0(38.0-42.0)^{\mathrm{b}}$ & $41.0(38.0-42.0)^{\mathrm{b}}$ \\
\hline$<37 \mathrm{~g} / \mathrm{l} \mathrm{N}(\%)$ & $13(59)$ & $7(32)$ & $6(27)$ & $3(21)$ & $14(47)$ & $6(20)$ & $2(7)$ & $3(16)$ \\
\hline Prealbumin (g/l) & $0.25(0.19-0.30)^{\mathrm{b}}$ & $0.20(0.16-0.21)^{\mathrm{a}}$ & $0.20(0.19-0.22)^{\mathrm{a}}$ & $0.20(0.18-0.0 .25)^{\mathrm{ab}}$ & $0.23(0.21-0.25)^{\mathrm{b}}$ & $0.18(0.17-0.21)^{\mathrm{a}}$ & $0.19(0.18-0.21)^{\mathrm{a}}$ & $0.19(0.18-0.22)^{\mathrm{a}}$ \\
\hline$<0.2 \mathrm{~g} / \mathrm{L} \mathrm{N}(\%)$ & $6(27)$ & $8(37)$ & $10(45)$ & $5(38)$ & $5(17)^{\mathrm{a}}$ & $17(57)^{\mathrm{b}}$ & $15(50)^{\mathrm{b}}$ & $10(52)^{\mathrm{b}}$ \\
\hline Calcium (mmol/l) & $2.29(2.24-2.37)$ & $2.39(2.33-2.43)$ & $2.37(2.28-2.39)$ & $2.31(2.26-2.39)$ & $2.31(2.24-2.38)$ & $2.37(2.31-2.44)$ & $2.31(2.28-2.38)$ & $2.33(2.31-2.38)$ \\
\hline 25(OH)-vitamin-D3 (ng/ml) & $13.0(10.0-23.0)^{\mathrm{a}}$ & I & $29.5(26.5-32.0)^{\mathrm{b}}$ & $27.0(22.0-29.0)^{\mathrm{b}}$ & $17.0(11.0-23.0)^{\mathrm{a}}$ & I & $26.9(22.5-30.5)^{\mathrm{b}}$ & $25.0(20.0-30.0)^{\mathrm{b}}$ \\
\hline$<30 \mathrm{ng} / \mathrm{ml} \mathrm{N}(\%)$ & $19(86)$ & I & $10(45)$ & $10(71)$ & $25(83)$ & l & $18(60)$ & $13(68)$ \\
\hline Parathyroid hormone (pg/ml) & $48.3(41.5-58.9)$ & I & I & $44.1(35.1-47.1)$ & $46.8(36.4-54.0)$ & I & I & $39.5(32.3-43.3)$ \\
\hline$>45 \mathrm{pg} / \mathrm{ml} \mathrm{N}(\%)$ & $13(59)$ & l & I & $6(43)$ & $15(50)$ & l & l & $4(21)$ \\
\hline Thiamin (nmol/l) & $157(150-174)$ & I & $193(155-193)$ & $197(174-215)$ & $147(134-175)$ & I & $177(158-191)$ & $181(149-218)$ \\
\hline$<126 \mathrm{nmol} / \mathrm{l} \mathrm{N}(\%)$ & $2(9)$ & / & $1(5)$ & $0(0)$ & $5(17)$ & I & $1(3)$ & $0(0)$ \\
\hline
\end{tabular}




\section{Erythrocyte folate (nmol/l)}

$<945 \mathrm{nmol} / \mathrm{l} \mathrm{N}(\%)$

Serum folate (nmol/l)

Vitamin B-12 (pmol/l)

$<140 \mathrm{pmol} / \mathrm{l} \mathrm{N}(\%)$

\section{7 (1023-1429) /}

4 (18)

$16.8(12.9-24.0)$

284 (209-334)

$1(5)$
1760 (1457-1961) 1940 (1421-2169)

2 (9)

$26.9(22.8-33.4) \quad 27.9(22.8-41.0)$

$252(227-345) \quad 221(195-278)$

$0(0)$

$1234(1036-1377)^{\mathrm{a}} \quad /$

5 (17)

$17.7(14.7-20.5)^{\mathrm{a}}$

293 (248-358)
$0(0)$

$22.8(18.4-28.4)^{\mathrm{b}} \quad 20.2(15.6-26.4)^{\mathrm{b}}$

$311(224-464) \quad 311(216-432)$

$0(0)$

${ }^{1}$ Labeled medians or percentages without a common letter differ between time-points for each surgical model, as tested by paired pairwise post hoc comparisons with Holm-Bonferroni correction or paired McNemar test. Normal ranges are as follows: hemoglobin [12-17] g/dl; ferritin

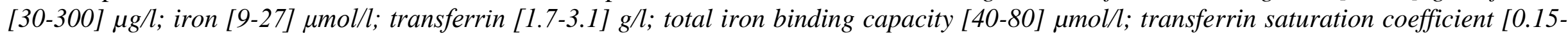
$0.35] \%$; albumin [37-50] g/l; prealbumin [0.2-0.35] g/l; calcium [2.1-2.65] mmol/l; 25(OH)-vitamin-D3 [30-100] ng/ml; thiamin [126-250] nmol/l; serum folate [7-39.5] nmol/l, vitamin B-12 [140-490] pmol/l. 


\section{FIGURE LEGENDS}

FIGURE 1. Changes in body composition in the GBP and SG groups at baseline (T0) and along the follow-up (T3, T6 and T12).

Results are expressed as means \pm SDs. Evolution of body composition during follow-up. Gastric sleeve in grey and GBP in black; top left panel fat free mass; top right panel total fat mass, low left panel appendicular fat free mass (i.e. arms + legs), low right panel appendicular fat mass. Labeled means without a common letter differ between time-points for each surgical model, as tested by paired pairwise post hoc comparisons with Holm-Bonferroni correction. No significant difference between GBP and SG was observed. 


\section{SuPPlemental Data}

SUPPLEMENTAL TABLE 1. PANDiet scores and probabilities of nutrient adequacy according to the surgical models at baseline, 3 months and 12 months (calculated from foods only) ${ }^{1}$

\begin{tabular}{|c|c|c|c|c|c|c|}
\hline & \multicolumn{3}{|c|}{ GBP } & \multicolumn{3}{|c|}{ SG } \\
\hline & $\begin{array}{c}\text { Baseline } \\
\mathrm{n}=22\end{array}$ & $\begin{array}{c}3 \text { months } \\
n=22\end{array}$ & $\begin{array}{c}12 \text { months } \\
n=14\end{array}$ & $\begin{array}{c}\text { Baseline } \\
\mathrm{n}=30\end{array}$ & $\begin{array}{c}3 \text { months } \\
n=30\end{array}$ & $\begin{array}{c}12 \text { months } \\
n=19\end{array}$ \\
\hline PANDiet & $66.0(60.7-70.5)^{\mathrm{b}}$ & $51.6(47.8-53.7)^{\mathrm{a}}$ & $52.1(46.1-57.6)^{\mathrm{a}}$ & $57.7(54.0-62.1)^{\mathrm{b}}$ & $47.6(40.9-53.0)^{\mathrm{a}}$ & $52.9(46.7-60.6)^{\mathrm{b}}$ \\
\hline Moderation Sub-score & $64.6(58.6-85.6)$ & $82.1(75.6-85.3)$ & $74.3(61.8-81.2)$ & $63.2(51.3-73.5)^{\mathrm{a}}$ & $70.9(63.8-80.0)^{\mathrm{b}}$ & $73.1(66.7-78.1)^{\mathrm{b}}$ \\
\hline Protein & $1.00(1.00-1.00)$ & $1.00(1.00-1.00)$ & $1.00(1.00-1.00)$ & $1.00(1.00-1.00)$ & $1.00(1.00-1.00)$ & $1.00(1.00-1.00)$ \\
\hline Total Carbohydrate & $1.00(1.00-1.00)$ & $1.00(0.97-1.00)$ & $1.00(0.98-1.00)$ & $1.00(1.00-1.00)$ & $1.00(1.00-1.00)$ & $1.00(1.00-1.00)$ \\
\hline Total Fat & $0.99(.035-1.00)$ & $0.81(0.58-0.93)$ & $0.59(0.05-0.95)$ & $0.84(0.52-0.96)$ & $0.42(0.02-0.84)$ & $0.57(0.05-0.89)$ \\
\hline SFA & $0.19(0.04-0.61)$ & $0.12(0.02-0.35)$ & $0.02(0.00-0.29)$ & $0.05(0.00-0.30)$ & $0.02(0.00-0.15)$ & $0.10(0.00-0.28)$ \\
\hline Cholesterol & $0.66(0.36-0.99)^{\mathrm{a}}$ & $0.99(0.94-1.00)^{\mathrm{b}}$ & $0.95(0.51-0.99)^{\mathrm{ab}}$ & $0.41(0.15-0.79)^{\mathrm{a}}$ & $0.98(0.65-1.00)^{\mathrm{b}}$ & $1.00(0.65-1.00)^{\mathrm{b}}$ \\
\hline Sodium & $0.43(0.18-0.77)^{\mathrm{a}}$ & $1.00(0.96-1.00)^{\mathrm{c}}$ & $0.97(0.71-1.00)^{\mathrm{b}}$ & $0.51(0.01-0.78)^{\mathrm{a}}$ & $1.00(0.97-1.00)^{\mathrm{b}}$ & $0.99(0.70-1.00)^{\mathrm{b}}$ \\
\hline Adequacy Sub-score & $60.8(53.0-72.0)^{\mathrm{b}}$ & $22.1(14.9-34.5)^{\mathrm{a}}$ & $30.3(22.8-42.8)^{\mathrm{a}}$ & $51.6(38.4-69.0)^{\mathrm{c}}$ & $20.6(11.9-35.2)^{\mathrm{a}}$ & $27.0(16.8-44.4)^{\mathrm{b}}$ \\
\hline Protein & $0.51(0.18-0.78)^{\mathrm{b}}$ & $0.00(0.00-0.03)^{\mathrm{a}}$ & $0.30(0.05-0.71)^{\mathrm{b}}$ & $0.47(0.17-0.81)^{\mathrm{c}}$ & $0.00(0.00-0.08)^{\mathrm{a}}$ & $0.05(0.00-0.68)^{\mathrm{b}}$ \\
\hline Total Carbohydrate & $0.86(0.20-0.98)$ & $0.43(0.04-0.82)$ & $0.20(0.01-0.62)$ & $0.44(0.03-0.68)$ & $0.09(0.00-0.75)$ & $0.24(0.00-0.52)$ \\
\hline Total Fat & $0.69(0.50-1.00)$ & $0.93(0.70-0.99)$ & $1.00(0.85-1.00)$ & $1.00(0.82-1.00)$ & $0.99(0.84-1.00)$ & $1.00(0.93-1.00)$ \\
\hline PUFA & $0.44(0.25-0.80)$ & $0.35(0.07-0.73)$ & $0.14(0.02-0.60)$ & $0.49(0.21-0.76)$ & $0.49(0.07-0.83)$ & $0.66(0.24-0.91)$ \\
\hline Fibre & $0.19(0.02-0.54)^{\mathrm{c}}$ & $0.00(0.00-0.00)^{\mathrm{a}}$ & $0.00(0.00-0.00)^{\mathrm{b}}$ & $0.02(0.00-0.06)^{\mathrm{c}}$ & $0.00(0.00-0.00)^{\mathrm{a}}$ & $0.00(0.00-0.00)^{\mathrm{b}}$ \\
\hline Vitamin A & $0.71(0.30-0.91)^{\mathrm{b}}$ & $0.12(0.00-0.51)^{\mathrm{a}}$ & $0.43(0.05-0.70)^{\mathrm{ab}}$ & $0.67(0.44-0.96)^{\mathrm{b}}$ & $0.07(0.00-0.61)^{\mathrm{a}}$ & $0.16(0.00-0.59)^{\mathrm{a}}$ \\
\hline Thiamin & $0.84(0.48-0.97)^{\mathrm{b}}$ & $0.01(0.00-0.19)^{\mathrm{a}}$ & $0.16(0.01-0.42)^{\mathrm{a}}$ & $0.56(0.31-0.77)^{\mathrm{b}}$ & $0.02(0.00-0.32)^{\mathrm{a}}$ & $0.01(0.00-0.35)^{\mathrm{a}}$ \\
\hline Riboflavin & $0.89(0.79-0.98)^{\mathrm{b}}$ & $0.06(0.01-0.67)^{\mathrm{a}}$ & $0.12(0.03-0.75)^{\mathrm{a}}$ & $0.83(0.57-0.93)^{\mathrm{b}}$ & $0.21(0.00-0.46)^{\mathrm{a}}$ & $0.08(0.00-0.70)^{\mathrm{a}}$ \\
\hline Niacin & $0.99(0.76-1.00)^{\mathrm{b}}$ & $0.09(0.00-0.60)^{\mathrm{a}}$ & $0.54(0.10-0.82)^{\mathrm{a}}$ & $0.93(0.85-0.99)^{\mathrm{b}}$ & $0.34(0.00-0.84)^{\mathrm{a}}$ & $0.68(0.41-0.98)^{\mathrm{a}}$ \\
\hline Vitamin B-6 & $0.81(0.54-0.98)^{\mathrm{b}}$ & $0.00(0.00-0.04)^{\mathrm{a}}$ & $0.00(0.00-0.28)^{\mathrm{a}}$ & $0.44(0.11-0.96)^{\mathrm{b}}$ & $0.00(0.00-0.10)^{\mathrm{a}}$ & $0.01(0.00-0.08)^{\mathrm{a}}$ \\
\hline Folate & $0.83(0.32-0.97)^{\mathrm{b}}$ & $0.04(0.01-0.17)^{\mathrm{a}}$ & $0.08(0.01-0.48)^{\mathrm{a}}$ & $0.56(0.30-0.81)^{\mathrm{b}}$ & $0.03(0.00-0.18)^{\mathrm{a}}$ & $0.04(0.01-0.16)^{\mathrm{a}}$ \\
\hline Vitamin B-12 & $0.88(0.75-0.98)^{\mathrm{b}}$ & $0.31(0.02-0.80)^{\mathrm{a}}$ & $0.72(0.38-0.90)^{\mathrm{b}}$ & $0.87(0.76-0.97)^{\mathrm{b}}$ & $0.63(0.12-0.83)^{\mathrm{a}}$ & $0.73(0.25-0.86)^{\mathrm{a}}$ \\
\hline
\end{tabular}




\begin{tabular}{llllllll} 
Vitamin C & $0.63(0.20-0.95)^{\mathrm{b}}$ & $0.05(0.00-0.57)^{\mathrm{ab}}$ & $0.09(0.00-0.47)^{\mathrm{a}}$ & $0.26(0.00-0.82)^{\mathrm{b}}$ & $0.00(0.00-0.16)^{\mathrm{a}}$ & $0.04(0.00-0.21)^{\mathrm{ab}}$ \\
Vitamin D & $0.00(0.00-0.10)^{\mathrm{b}}$ & $0.00(0.00-0.00)^{\mathrm{a}}$ & $0.00(0.00-0.02)^{\mathrm{ab}}$ & $0.02(0.00-0.30)$ & $0.00(0.00-0.08)$ & $0.15(0.00-0.44)$ \\
Vitamin E & $0.29(0.08-0.89)^{\mathrm{c}}$ & $0.00(0.00-0.00)^{\mathrm{a}}$ & $0.00(0.00-0.03)^{\mathrm{b}}$ & $0.18(0.02-0.44)^{\mathrm{b}}$ & $0.00(0.00-0.01)^{\mathrm{a}}$ & $0.13(0.00-0.39)^{\mathrm{b}}$ \\
Calcium & $0.87(0.07-0.97)^{\mathrm{b}}$ & $0.28(0.00-0.77)^{\mathrm{a}}$ & $0.49(0.07-0.86)^{\mathrm{a}}$ & $0.82(0.43-0.97)^{\mathrm{b}}$ & $0.06(0.00-0.35)^{\mathrm{a}}$ & $0.08(0.00-0.56)^{\mathrm{a}}$ \\
Magnesium & $0.00(0.00-0.00)^{\mathrm{b}}$ & $0.00(0.00-0.00)^{\mathrm{a}}$ & $0.00(0.00-0.00)^{\mathrm{a}}$ & $0.00(0.00-0.00)^{\mathrm{b}}$ & $0.00(0.00-0.00)^{\mathrm{a}}$ & $0.00(0.00-0.00)^{\mathrm{a}}$ \\
Zinc & $0.72(0.35-0.90)^{\mathrm{b}}$ & $0.01(0.00-0.14)^{\mathrm{a}}$ & $0.03(0.01-0.21)^{\mathrm{a}}$ & $0.58(0.27-0.86)^{\mathrm{c}}$ & $0.01(0.00-0.06)^{\mathrm{a}}$ & $0.11(0.00-0.51)^{\mathrm{b}}$ \\
Phosphorus & $1.00(1.00-1.00)^{\mathrm{c}}$ & $0.59(0.03-0.96)^{\mathrm{a}}$ & $0.90(0.78-0.99)^{\mathrm{b}}$ & $0.99(0.96-1.00)^{\mathrm{c}}$ & $0.60(0.04-0.87)^{\mathrm{a}}$ & $0.86(0.14-1.00)^{\mathrm{b}}$ \\
Potassium & $0.69(0.45-0.93)^{\mathrm{b}}$ & $0.01(0.00-0.29)^{\mathrm{a}}$ & $0.01(0.00-0.15)^{\mathrm{a}}$ & $0.39(0.14-0.89)^{\mathrm{c}}$ & $0.00(0.00-0.02)^{\mathrm{a}}$ & $0.03(0.00-0.12)^{\mathrm{b}}$ \\
Iron & $0.85(0.65-1.00)^{\mathrm{b}}$ & $0.04(0.00-0.55)^{\mathrm{a}}$ & $0.25(0.00-0.55)^{\mathrm{a}}$ & $0.93(0.55-1.00)^{\mathrm{b}}$ & $0.10(0.00-0.45)^{\mathrm{a}}$ & $0.15(0.00-0.85)^{\mathrm{a}}$ \\
\hline
\end{tabular}

${ }^{1}$ Labeled medians without a common letter differ between time-points for each surgical model, as tested by paired pairwise post hoc comparisons with Holm-Bonferroni correction. 
